Pacific

Journal of

Mathematics

CONFORMALLY INVARIANT NON-LOCAL OPERATORS

Thomas Branson and A. Rod Gover 


\title{
CONFORMALLY INVARIANT NON-LOCAL OPERATORS
}

\author{
Thomas Branson and A. Rod Gover
}

\begin{abstract}
On a conformal manifold with boundary, we construct conformally invariant local boundary conditions $B$ for the conformally invariant power of the Laplacian $\square_{k}$, with the property that $\left(\square_{k}, B\right)$ is formally self-adjoint. These boundary problems are used to construct conformally invariant nonlocal operators on the boundary $\Sigma$, generalizing the conformal Dirichlet-to-Robin operator, with principal parts which are odd powers $h$ (not necessarily positive) of $\left(-\Delta_{\Sigma}\right)^{1 / 2}$, where $\Delta_{\Sigma}$ is the boundary Laplace operator. The constructions use tools from a conformally invariant calculus.
\end{abstract}

\section{Introduction.}

Conformally invariant differential operators have long been important in Physics. Since null geodesics depend only on conformal structure, the classical field equations describing massless particles, for example the Maxwell and Dirac (neutrino) equations, can be expected to depend only on conformal structure $[4, \mathbf{2 0}]$. In recent years, much work has been done on the systematic construction, understanding, and classification of conformally invariant differential operators $[2,3,6,7,21,23,28,30,31,41,45,47]$. In the light of string theory and other recent developments [19], the importance of such operators, even beyond those familiar from earlier physical investigations, has been underlined. New applications to natural geometric variational principles, and physical least action principles $[\mathbf{2 4}, \mathbf{3 8}, \mathbf{3 9}, \mathbf{4 0}]$, have shown the importance of non-local objects like pseudo-differential operators and values and residues of spectral zeta and eta functions, and of boundary value problems.

The construction and classification problems for conformally invariant boundary value problems, and for conformally invariant pseudo-differential operators, are quite underdeveloped in comparison to the corresponding problems for conformally invariant differential operators. Yet there are natural points of departure for investigations into such questions. For example, much of the recent work on invariant differential operators has centered conceptually on the corresponding construction of homorphisms between Verma modules and their generalizations, a central group representation theoretic problem. The related notion of Harish-Chandra modules and the theory of 
the Knapp-Stein intertwining operators and their analytic continuation indicate that it is reasonable to expect analogous invariant operators of all real orders.

For example, the operators

$$
A_{2 r}=\frac{\Gamma\left(B+\frac{r}{2}+\frac{1}{2}\right)}{\Gamma\left(B-\frac{r}{2}+\frac{1}{2}\right)}, \quad \text { where } B=\sqrt{-\Delta+\left(\frac{n-2}{2}\right)^{2}},
$$

are intertwining for the spherical principal series of $\mathrm{SO}_{0}(n, 1)$. This is another way of saying that they are conformally invariant, when viewed as acting between density bundles of the appropriate weights, under the conformal transformation group of the standard sphere $S^{n-1}$. The differential operators among these are the cases $r=0,2,4, \ldots$; the question of their generalization to conformally invariant operators on arbitrarily curved manifolds has been of much recent interest [31]. The case $r=1$, in which the operator is simply $B$, is also a familar object: the conformal Dirichlet-toRobin operator on $S^{n}$. The generalization $\mathcal{B}$ of this operator to arbitrarily curved conformal structures is the most elementary case of the series of operators we construct in our main theorems.

The operator $\mathcal{B}$ is closely related to a fundamental object in the subject of Electrical Impedance Tomography, where the basic problem is reconstruction of a conformal factor on a manifold $M$ from the Dirichlet-to-Neumann operator on its boundary $\Sigma[35,46]$. The conformal factor represents the density of, for example, a human head, while the Dirichlet-to-Neumann operator represents a response, measured on the skin, to applied current. As a practical matter, one attaches electrodes to obtain a discrete approximation to this continuous problem.

The above considerations raise natural questions as to the possible existence of arbitrarily curved generalizations of the $A_{2 r}$ for $2 r=1,3, \ldots$, and even for arbitrary $r$. The question of arbitrary $r$ is the subject of [43], where the existence of such generalizations is shown, modulo an obstruction at certain orders in even dimensions first identified in $[\mathbf{2 6}, \mathbf{3 1}]$. In [43] the main algebraic objects are asymptotic expansions of pseudo-differential symbols; these are then "promoted" to operators which exist, and are invariant, modulo operators of order $-\infty$.

The present paper is concerned with operators of odd integer order which can be described exactly in terms of boundary value problems. Such operators have already shown their importance in the theory of extremals of the functional determinant, a fundamental object in String Theory [13, 17]. These series of operators are closely related to series of harmonic analytic sharp inequalities of Sobolev imbedding and Moser-Trudinger type (see $[\mathbf{5}, \mathbf{1 1}, \mathbf{1 6}, \mathbf{1 8}]$ for manifolds without boundary, and $[\mathbf{1 7}, \mathbf{2 4}, \mathbf{2 5}]$ for manifolds with boundary). The relation to such objects indicates a deep 
connection to geometric information that generalizes, but should go significantly beyond, the information used in the Yamabe problem, where only one Sobolev imbedding is involved.

The authors would like to thank Peter Gilkey, C. Robin Graham and Gerd Grubb for enlightening correspondence. A.R.G. also thanks the Mathematics Department of the University of Iowa for its hospitality during AugustSeptember of 1997, when the core of this work was done.

\section{Background.}

The conformally invariant Dirichlet-to-Robin operator (see e.g., [10], Example 1.3) on the boundary to a manifold is constructed in two steps. The first is to extend a density of an appropriate weight off the boundary so that the resulting density of the same weight on the interior is in the kernel of the conformally invariant Laplace operator. The second step is to apply to this density, at the boundary, an invariant first order normal derivative operator obtained by adding a multiple of the mean curvature of the boundary imbedding. Such an operator is sometimes called a Robin operator. The result is a first order pseudo-differential operator on the boundary which by construction is conformally invariant, and which has the form $\left(-\Delta_{\Sigma}\right)^{1 / 2}+$ (lower order terms).

In this article we produce a family of higher order, and negative order, analogues of the Dirichlet-to-Robin operator. We explicitly construct conformally invariant, formally self-adjoint pseudo-differential operators whose principal parts are (positive and negative) odd integer powers $h$ of $\left(-\Delta_{\Sigma}\right)^{1 / 2}$. For even dimensional boundaries we obtain such operators for all odd integers $h$, while for odd dimensional boundaries of dimension $n^{\Sigma} \geq 3$, the construction works for odd integers in the range $|h| \leq n^{\Sigma}-2$. These results are given in Theorems 8.1, 8.4, and 8.5. We believe that the odd $n^{\Sigma}$ range can eventually be improved to $|h| \leq n^{\Sigma}$. For example, for $n^{\Sigma}=3$, the existence of a third order invariant operator with the correct leading symbol follows from the construction used in our theorems, given the construction in [17] of local boundary conditions for an invariant fourth-order operator.

A conformal manifold is a pair $(M,[g])$ where $M$ is a smooth manifold and $[g]$ is a conformal equivalence class of metrics. Two metrics $g$ and $\widehat{g}$ are said to be conformally equivalent, or just conformal, if $\widehat{g}$ is a positive scalar function multiple of $g$. In this case it is convenient to write $\widehat{g}=\Omega^{2} g$ for some positive smooth function $\Omega$. (The transformation $g \mapsto \widehat{g}$, which changes the choice of metric from the conformal class, is termed a conformal rescaling.) For present purposes (until elliptic theory comes into play), we shall allow the metrics in the equivalence class to have any fixed signature.

For $n \geq 3$ we will work with conformal $n$-manifolds with boundary $\partial M=\Sigma$ and throughout, without further comment, we will assume that 
the conformal structure extends smoothly to a collar of the boundary. Our results will not depend on the choice of extension. We simply need that the conformal metric is nondegenerate at the boundary. For the purposes of constructing differential operators on the boundary we can thus view the boundary as an embedded hypersurface in the extended conformal manifold. In Section 4 we introduce a conformally invariant calculus for dealing with hypersurfaces in a conformal manifold. This is applied immediately in Section 5 to construct series of conformally invariant boundary operators. The results are presented in Theorem 5.1; to our knowledge this is the first time such operators have been constructed for the cases of order greater than 3. In Section 7 it is shown that the boundary operators can be adjusted to yield, in combination with the interior operators (as described in Proposition 4.1), formally self-adjoint boundary problems. With the observation that our boundary problems are also properly elliptic and satisfy the Lopatinski-Shapiro conditions (as discussed in Section 6) we are able to perform the main construction in Section 8.

We will write $\mathcal{E}$ for the sheaf of germs of smooth functions on $M$. In line with this we will often write $\mathcal{E}^{a}$ and $\mathcal{E}_{a}$ for, respectively, the tangent and cotangent bundles to $M$ (which we will not distinguish from the respective sheaves of germs of smooth sections). Tensor products of these bundles will be indicated by adorning the symbol $\mathcal{E}$ with appropriate indices. For example, in this notation $\otimes^{2} T^{*} M$ is written $\mathcal{E}_{a b}$. The pairing of vectors with their duals and the generalization of this to tensors will be indicated by repeated indices in the usual fashion. Unless otherwise indicated, our indices will be abstract indices in the sense of Penrose [42]. An index which appears twice, once raised and once lowered, indicates a contraction. In case a frame is chosen and the indices are concrete, use of the Einstein summation convention (to implement the contraction) is understood. Given a choice of metric, indices will be raised and lowered using the metric without explicit mention.

For purposes of calculating and producing explicit formulae it is often useful to choose, arbitrarily, a metric from the conformal equivalence class. Then the manifold becomes equipped with the canonical Levi-Civita connection $\nabla_{a}$. This is the unique torsion free connection on the tangent space and its tensor powers which preserves the chosen metric. The curvature $R_{a b}{ }^{c} d$ of this connection is known as the Riemannian curvature, and is defined by

$$
\left(\nabla_{a} \nabla_{b}-\nabla_{b} \nabla_{a}\right) v^{c}=R_{a b}^{c} v^{d} .
$$

It is useful to observe that $R_{a b c d}$ can be decomposed into the totally tracefree Weyl curvature $C_{a b c d}$ and a remaining part described by the symmetric Rho-tensor $\mathrm{P}_{a b}$, according to

$$
R_{a b c d}=C_{a b c d}+2 g_{c[a} \mathrm{P}_{b] d}+2 g_{d[b} \mathrm{P}_{a] c},
$$


where $[\cdots]$ indicates the skew part over the enclosed indices. The Rho-tensor is a trace modification of the Ricci tensor $R_{a b}$.

We define the density bundles $\mathcal{E}[w]$ on $M$ as follows. The bundle whose smooth sections are metrics from the conformal class is a ray subbundle (i.e., a fibre subbundle with fibre $\mathbb{R}_{+}$) of $\mathcal{E}_{a b}$. We identify this subbundle with a ray bundle of scalars, which we denote $\mathcal{E}_{+}[-2]$. For each $w \in \mathbb{R}$ the ray bundle $\mathcal{E}_{+}[w]$ is then defined to be the $\left(-\frac{w}{2}\right)^{\text {th }}$ power of $\mathcal{E}_{+}[-2]$. These each canonically extend to a line bundle $\mathcal{E}[w]$ with a distinguished positive axis in each fibre. Given a choice of metric, the Levi-Civita connection determines a connection on tensor densities; for example, on $\mathcal{E}^{a}[w]=\mathcal{E}[w] \otimes \mathcal{E}^{a}$. Of course, this connection is not invariant under conformal rescaling, but transforms by well-known formulae (see for example $[\mathbf{1}, \mathbf{4 2}]$ ).

\section{Hypersurfaces and operators.}

Let $(M,[g])$ be a conformal manifold of dimension $n$. Suppose that $\Sigma$ is a hypersurface in $M$, that is $\Sigma$ is a dimension $n^{\Sigma}=n-1$ imbedded submanifold of $M$. We shall assume further that in the case of indefinite metrics, the normal to the hypersurface has nowhere vanishing length. The tangent bundle to $T \Sigma$ to $\Sigma$ is naturally a subbundle of $\left.T M\right|_{\Sigma}$. Via the inclusion $i: \Sigma \rightarrow M, \Sigma$ inherits a natural conformal structure: For each metric $g$ from the conformal class $[g]$ of metrics on $M, g^{\Sigma}:=i^{*} g$ is a metric on $\Sigma$. If $\widehat{g}=\Omega^{2} g$, then clearly $\widehat{g^{\Sigma}}=i^{*} \widehat{g}=\left(i^{*} \Omega\right)^{2} g^{\Sigma}$.

The sheaf of germs of smooth functions on $\Sigma$ will be denoted by $\mathcal{E}_{\Sigma}$. Since any section of this may be extended to a smooth function on $M$, we have $\mathcal{E}_{\Sigma}=\left.\mathcal{E}\right|_{\Sigma}$. Similarly it is clear that $\mathcal{E}_{\Sigma}[w]=\left.\mathcal{E}[w]\right|_{\Sigma}$, where $\mathcal{E}_{\Sigma}[w]$ denotes the bundle of densities of weight $w$ and intrinsic to $\Sigma$ as a conformal manifold.

We observed that $T \Sigma$ is a subbundle of $\left.T M\right|_{\Sigma}$. In fact the conformal structure enables us to split $\left.T M\right|_{\Sigma}$ into a direct sum of $T \Sigma$ with the normal bundle $\mathcal{N}^{a}$. Let $N^{a}$ be a unit normal field to $\Sigma$. Note that this is a section, over $\Sigma$, of $\mathcal{E}^{a}[-1]$.

We shall take $N^{a}$ to be extended as a unit section of $\mathcal{E}^{a}[-1]$ over a neighbourhood of $\Sigma$. Then, over $\Sigma$, the map

$$
\left.T M\right|_{\Sigma} \cong T \Sigma \oplus \mathcal{N}^{a}
$$

is given by

$$
v^{a} \mapsto\left(v^{a}-N^{a} N_{b} v^{b}\right)+N^{a} N_{b} v^{b}
$$

for $v^{a} \in \Gamma(T M)$. Since we shall always treat $T \Sigma$ as a subbundle of $\left.T M\right|_{\Sigma}$ in this way, it is reasonable to use the notation $\mathcal{E}_{\Sigma}^{a}$ for $T \Sigma$. Thus in this notation we have $\left.\mathcal{E}^{a}\right|_{\Sigma}=\mathcal{E}_{\Sigma}^{a} \oplus \mathcal{N}^{a}$.

In general with constructions of the above type, we shall write $\Sigma$ as a superscript or subscript, whichever is convenient. 
Given bundles $\mathcal{F}$ and $\mathcal{G}$ and a differential operator $B: \mathcal{F} \rightarrow \mathcal{G}$ on $M$, we shall need a notion of the normal order of $B$. Let the order of $B$ be $r$ and suppose that, in a neighbourhood of a point $p, \Sigma$ is given by the vanishing of a defining function $\sigma$. We say that $B$ has normal order $r_{N}$ at $p$ if there exists a section $\phi$ of $\mathcal{F}$ such that $B\left(\sigma^{r_{N}} \phi\right)(p) \neq 0$ but for any section $\phi^{\prime}$ of $\mathcal{F}$, $B\left(\sigma^{r_{N}+1} \phi^{\prime}\right)(p)=0$. Of course $r_{N} \leq r$, and is independent of the particular choice of defining function $\sigma$. An operator which has normal order $r_{N}$ at each $p \in \Sigma$ will be said to have normal order $r_{N}$. Note that the differential operators which are intrinsic to $\Sigma$ (those that only involve differentiation in directions within the hypersurface) have normal order $r_{N}=0$. On the other hand the normal derivative $N^{a} \nabla_{a}$ has normal order 1 on $\Sigma$.

Recall that on $M$ there is a series of conformally invariant differential operators with principal part a power of the Laplacian,

$$
\square_{k}: \mathcal{E}\left[\frac{k-n}{2}\right] \rightarrow \mathcal{E}\left[-\frac{k+n}{2}\right], \quad\left\{\begin{array}{l}
k=2,4, \ldots, n(n \text { even }), \\
k=2,4, \ldots(n \text { odd }),
\end{array}\right.
$$

where

$$
\square_{k} \phi=\Delta^{k / 2} \phi+\text { lower order terms. }
$$

(As mentioned above $\Delta$ is the usual Laplacian, i.e., $\Delta=\nabla^{a} \nabla_{a}$.) The existence of such operators in general is due to [31], although the order $k=2$ case is the usual Yamabe operator while the $k=4$ case is due to Paneitz $[41]$ and, later but independently, $[44,23]$. Before the general result was known, the $k=6$ case was also settled in [47]. In the next section we give an explicit formula for these (except for the $k=n$ cases) using the tractor-D operator (as in [1]).

On the other hand, Theorem 5.1 below describes a family of conformally invariant differential operators $\delta_{\ell}$ carrying densities (or, more generally, weighted tractors) on $M$ to similar objects on $\Sigma$. These, or their modifications as developed in Section 6 (see the proof of Proposition 7.1), may be composed with conformally invariant operators implementing extension from the boundary, via solution of appropriate elliptic boundary value problems, to yield conformally invariant operators on the boundary; this is Theorem 8.1. This construction is analogous to (and generalizes) that of the conformal Dirichlet-to-Neumann operator.

\section{Hypersurface tractors.}

On a conformal $n$-manifold, $n \geq 3$, there is a conformally invariant calculus based on the so-called tractor bundle. We shall briefly review this here as it underlies the construction of the invariant operators of Theorem 5.1 and Proposition 4.1 below. Further details can be found in $[\mathbf{1}, \mathbf{2 1}, \mathbf{3 0}]$. In fact we shall also need a version of this calculus on the 2-dimensional boundary manifold to a conformal 3-manifold. Thus the final part of this section is 
concerned with developing a tractor calculus for hypersurfaces in conformal 3-manifolds. Throughout this section, the conformal structure may have any fixed signature.

For a given choice of metric $g$, the tractor bundle $\mathcal{E}^{A}$ may be identified with a direct sum

$$
\left[\mathcal{E}^{A}\right]_{g}=\mathcal{E}[1] \oplus \mathcal{E}^{a}[-1] \oplus \mathcal{E}[-1] .
$$

If $U^{A}$ is a section of $\mathcal{E}^{A}$, we can thus write

$$
\left[U^{A}\right]_{g}=\left(\begin{array}{c}
\sigma \\
\mu^{a} \\
\rho
\end{array}\right) \in\left[\Gamma \mathcal{E}^{A}\right]_{g} .
$$

Under conformal rescaling $g \mapsto \hat{g}=\Omega^{2} g$ these quantities transform according to

$$
\left[U^{A}\right]_{\widehat{g}}=\left(\begin{array}{c}
\widehat{\sigma} \\
\widehat{\mu}^{a} \\
\widehat{\rho}
\end{array}\right)=\left(\begin{array}{c}
\sigma \\
\mu^{a}+\Upsilon^{a} \sigma \\
\rho-\Upsilon_{b} \mu^{b}-\frac{1}{2} \Upsilon^{b} \Upsilon_{b} \sigma
\end{array}\right),
$$

where $\Upsilon_{a}:=\Omega^{-1} \nabla_{a} \Omega$. In analogy with the term tensor (in differential geometry), we shall also describe any tensor product of the tractor bundle and its dual, and indeed invariant subbundles of these, as tractor bundles. If such a bundle is tensored with some bundle of densities $\mathcal{E}[w]$ then we shall describe the result as a weighted tractor bundle. We write $\mathcal{E}_{A B}[w]$ for $\mathcal{E}_{A} \otimes \mathcal{E}_{B} \otimes \mathcal{E}[w]$, and so on. In many cases we wish to indicate a weighted tractor bundle without being specific about the indices of the bundle or any symmetry these may possess. Thus we write $\mathcal{E}^{*}[w]$ to mean a weighted tractor bundle which is the tensor product of $\mathcal{E}[w]$ with any tractor bundle. Finally, repeated tractor indices indicate a contraction, just as for tensor indices.

The invariant projection $\mathcal{E}^{A} \rightarrow \mathcal{E}[1]$ may be regarded as a preferred element $X_{A} \in \Gamma \mathcal{E}_{A}[1]$ so that, with $U^{A}$ again as above, we have $\sigma=U^{A} X_{A}$. It also describes the invariant injection $\mathcal{E}[-1] \rightarrow \mathcal{E}_{A}$ according to $\rho \mapsto \rho X_{A}$. This tautological tractor $X_{A}$ is given, for any choice of metric $g$ from the conformal class, by $\left[X_{A}\right]_{g}=\left(\begin{array}{lll}1 & 0 & 0\end{array}\right)$.

The bundle $\mathcal{E}^{A}$ carries an invariant nondegenerate tractor metric $h_{A B}$, and a connection $\nabla_{a}$ which preserves this. If the signature of the underlying conformal structure is $(p, q)$, then the signature of $h_{A B}$ is $(p+1, q+1)$. For $U^{A}$ as above, this metric is given by $h_{A B} U^{A} U^{B}=2 \rho \sigma+\mu^{a} \mu_{a}$. The tractor metric will be used to raise and lower indices without further mention. The connection is given explicitly by the following formula for $\left[\nabla_{a} U^{B}\right]_{g}$ :

$$
\nabla_{a}\left(\begin{array}{c}
\sigma \\
\mu^{b} \\
\rho
\end{array}\right)=\left(\begin{array}{c}
\nabla_{a} \sigma-\mu_{a} \\
\nabla_{a} \mu^{b}+\delta_{a}^{b} \rho+\mathrm{P}_{a}^{b} \sigma \\
\nabla_{a} \rho-\mathrm{P}_{a c} \mu^{c}
\end{array}\right) .
$$


Of course this may be extended to a connection on any tractor bundle in the obvious way. (In fact, these connections are all induced connections of the canonical Cartan conformal connection.) The use of the same symbol $\nabla$ as for the Levi-Civita connection is no accident. In fact, more generally, we shall use $\nabla$ to mean the coupled Levi-Civita-tractor connection. This enables us, for example, to apply $\nabla$ to weighted tractor bundles, although in this case it is not conformally invariant of course. However, in terms of this coupled connection, one may construct an invariant second order operator between weighted tractor bundles,

$$
D_{A}: \mathcal{E}^{*}[w] \rightarrow \mathcal{E}^{*}[w-1] .
$$

This is called the tractor- $D$ operator and is given explicitly by

$$
D_{A} f=(n+2 w-2) \tilde{D}_{A} f-X_{A} \square f
$$

where

$$
\left[\tilde{D}^{A} f\right]_{g}:=\left(\begin{array}{c}
w f \\
\nabla^{a} f \\
0
\end{array}\right) \text { and } \square f:=\tilde{D}_{B} \tilde{D}^{B} f=\nabla_{b} \nabla^{b} f+w \mathrm{P} f
$$

(Note that $\tilde{D}^{A} f$ and $\square f$ are not separately invariant.)

Using these definitions, it is an elementary exercise to show that if $f \in$ $\Gamma(\mathcal{E}[w])$ then

$$
X_{A} D^{A} f=w(n+2 w-2) f
$$

and

$$
D^{A} X_{A} f=(n+2 w+2)(n+w) f .
$$

Using this and the definition of $D_{A}$ once more, one can obtain the following explicit construction of conformally invariant powers of the Laplacian (first observed by Eastwood [22]; see also [30]).

Proposition 4.1. The operators

$$
\square_{k}: \mathcal{E}^{*}\left[\frac{k-n}{2}\right] \rightarrow \mathcal{E}^{*}\left[-\frac{k+n}{2}\right], \text { where } k \geq 2 \text { is even, }
$$

defined by

$$
\square_{k}:=D^{A} \cdots D^{B} \square \underbrace{D_{B} \cdots D_{A}}_{(k-2) / 2}
$$

are conformally invariant differential operators of the form (up to a non-zero constant scale factor)

$$
\Delta^{k / 2}+\text { lower order terms }
$$

except when $n$ is even and $n \leq k$. 
Note that this proposition gives an explicit formula for these operators, although to rewrite this in terms of the Levi-Civita connection and its curvature is extremely tedious and not practical except in the lowest order cases. We should also warn the reader at this point that while the operators $\square_{k}$ are sometimes called "conformally invariant powers of the Laplacian", they are distinct from the operators $\Delta^{k / 2}$ and $\square^{k / 2}$. Indeed, the $\square^{k / 2}$ are not conformally invariant when $k>2$.

Let us now return to the discussion of a hypersurface $\Sigma$ in an $n$-manifold $M$ as in the previous section. In this case there are two settings for building a tractor calculus as described above. There is the ambient conformal manifold $M$, and there is also the hypersurface $\Sigma$ regarded as an intrinsic conformal $(n-1)$-manifold. Since the tractor bundle on $\Sigma$ will have fibres of dimension $n+1$ as compared to the fibre dimension $n+2$ of the ambient tractor bundle $\mathcal{E}^{A}$, we should use different indices for the tractor bundle of $\Sigma$. However, there is another approach that we shall adopt instead: We shall view the intrinsic tractor bundle on $\Sigma$ as a subbundle of the ambient tractor bundle restricted to $\Sigma,\left.\mathcal{E}^{A}\right|_{\Sigma}$. This is analogous to, and consistent with, our treatment of the tangent bundle $\mathcal{E}_{\Sigma}^{a}$ to $\Sigma$, as described above. First of all observe that along $\Sigma$ (and in all dimensions $n \geq 3$ ) there is a natural conformally invariant normal tractor (see [1]) $N^{A} \in \Gamma\left(\left.\mathcal{E}^{A}\right|_{\Sigma}\right)$, given in any metric $g$ by

$$
\left[N^{A}\right]_{g}:=\left(\begin{array}{c}
0 \\
N^{a} \\
-H
\end{array}\right),
$$

where $H=(n-1)^{-1} \nabla_{a} N^{a}$ is the mean curvature of $\Sigma$. (We will later take $\Sigma$ to be the boundary of a conformal manifold with positive definite metric signature, and we will choose the inward unit normal $N$. This convention makes the above-defined mean curvature negative on the standard sphere viewed as the boundary of the standard ball.)

Now under a conformal rescaling, $g \mapsto \widehat{g}=\Omega^{2} g, H$ transforms to $\widehat{H}=$ $H+N^{a} \Upsilon_{a}$. (Note that $H$ is being viewed as a $(-1)$-density.) Using this it is easy to see that there is a preferred class of metrics within the conformal class which have $H=0$ along $\Sigma$. For rescalings amongst metrics in this preferred class, $N^{a} \Upsilon_{a}=0$. If $n \geq 4$ then $n^{\Sigma} \geq 3$ and so the manifold $\Sigma$ has its own intrinsic tractor bundles, connections and so forth. In these cases, restricting to metrics from the preferred class, it is easily verified that the intrinsic tractor bundle to $\Sigma$ may be identified with the conformally invariant subbundle $\mathcal{E}_{\Sigma}^{A}$ of $\left.\mathcal{E}^{A}\right|_{\Sigma}$ which is orthogonal to the normal tractor $N^{A}$. Thus we have an invariant splitting

$$
\left.\mathcal{E}^{A}\right|_{\Sigma}=\mathcal{E}_{\Sigma}^{A} \oplus \mathcal{N}^{A}
$$

given by

$$
v^{A} \mapsto\left(v^{A}-N^{A} N_{B} v^{B}\right)+N^{A} N_{B} v^{B} .
$$


for $v^{A} \in \Gamma\left(\mathcal{E}^{A}\right)$. Of course this generalizes easily to tensor products of these bundles, and we shall always view the intrinsic tractor bundles of $\Sigma$ in this way; that is, as subbundles of the restrictions to $\Sigma$ of ambient tractor bundles, the sections of which are completely orthogonal to $N^{A}$. As a result, we need only one type of tractor index. Of course once we have identified any intrinsic tractor sections with sections of such subbundles, we need no longer restrict to the special class of metrics with $H=0$, as orthogonality to $N^{A}$ is a conformally invariant condition. We shall use the symbol $P_{\Sigma}$ to indicate the orthogonal projection from any ambient weighted tractor bundle, restricted to $\Sigma$, to the corresponding intrinsic-to- $\Sigma$ weighted tractor bundle. For example, $P_{\Sigma}\left(\left.\mathcal{E}^{A}\right|_{\Sigma}\right)=\mathcal{E}_{\Sigma}^{A}$. In fact we shall henceforth drop the explicit restriction to $\Sigma$ and regard this as implicit in the definition of $P_{\Sigma}$. Thus we shall write, for example, $P_{\Sigma}\left(\mathcal{E}_{A B}[w]\right)=\mathcal{E}_{A B}^{\Sigma}[w]$; any section $f_{A B}$ of this bundle has the property that $f_{A B} N^{A}=0=f_{A B} N^{B}$. For the cases $n^{\Sigma} \geq 3$ the intrinsic-to- $\Sigma$ tractor-D operator will be denoted $D_{A}^{\Sigma}$, and similarly the tautological tractor belonging to $\Sigma$ will be denoted $X_{A}^{\Sigma}$.

According to the definitions of $X_{A}$ and $N^{A}$, we have $X_{A} N^{A}=0$. Thus $P_{\Sigma}\left(X_{A}\right)=\left.X_{A}\right|_{\Sigma}$, and it follows from the definition of $X_{A}^{\Sigma}$ that in fact $X_{A}^{\Sigma}=\left.X_{A}\right|_{\Sigma}$. If $n \geq 4$, then it follows immediately from these observations and (5) that if $f \in \mathcal{E}_{\Sigma}^{*}[w]$, then, on $\Sigma$,

$$
D_{\Sigma}^{A} X_{A} f=\left(n^{\Sigma}+2 w+2\right)\left(n^{\Sigma}+w\right) f=(n+2 w+1)(n+w-1) f .
$$

As mentioned above, we also wish to treat the $n=3, n^{\Sigma}=2$ case. On general 2-dimensional conformal manifolds, one does not expect a natural tractor calculus analogous to that on the higher dimensional structures (owing to the vastly different local structure in this case). However, 2dimensional hypersurfaces inherit extra local structure from the ambient 3-manifold. As we now show, this rigidity enables the construction of a natural tractor calculus on such hypersurfaces. For the remainder of this section let $n=3$.

We define the tractor bundle $\mathcal{E}_{\Sigma}^{A}$ on $\Sigma$ to be the subundle of $\left.\mathcal{E}^{A}\right|_{\Sigma}$ which is orthogonal to $N^{A}$. Thus in this case we get a splitting $\left.\mathcal{E}^{A}\right|_{\Sigma}=\mathcal{E}_{\Sigma}^{A} \oplus \mathcal{N}^{A}$, as in the higher dimensional cases. Now a tractor connection $\nabla^{\Sigma}$ on this is induced from the ambient tractor connection, $\nabla$, in the obvious way. Viz for $v^{A} \in \Gamma \mathcal{E}^{A}$ we take

$$
\nabla_{a}^{\Sigma} v^{B}:=P_{\Sigma} \nabla_{a} v^{B}
$$

where, on the right-hand-side $v^{B}$ is a section of $\mathcal{E}^{A}$ in a neighbourhood of $\Sigma$ that agrees with $v^{B}$ over $\Sigma$ and $P_{\Sigma}$ indicates the projection

$$
P_{\Sigma} \nabla_{a} v^{B}=\left(\delta_{a}^{a^{\prime}}-N^{a^{\prime}} N_{a}\right)\left(\delta_{B^{\prime}}^{B}-N^{B} N_{B^{\prime}}\right) \nabla_{a^{\prime}} v^{B^{\prime}} .
$$

It is easily verified that $\nabla^{\Sigma}$ is independent of any choices of extensions off $\Sigma$ and satisfies the required Leibniz rule. 
Now, for $f$, a section of a weighted intrinsic tractor bundle (i.e., a section of some tensor product involving $\mathcal{E}_{\Sigma}^{A}$, its dual and $\mathcal{E}_{\Sigma}[w]$ or a section of a subbundle of such a bundle) define $\tilde{D}_{A}^{\Sigma} f$ by

$$
\tilde{D}_{A}^{\Sigma} f:=P_{\Sigma} \tilde{D}_{A} f
$$

where, on the right hand side, $f$ has been extended to a section of a tractor bundle in a neighbourhood of $\Sigma$. Then, in a choice of conformal scale such that $H=0$, we have

$$
\left[\tilde{D}_{\Sigma}^{A} f\right]_{g}:=\left(\begin{array}{c}
w f \\
\nabla_{\Sigma}^{a} f \\
0
\end{array}\right),
$$

and so we have formal agreement with the higher dimensional cases. The operator $D_{A P}^{\Sigma}:=2 P_{\Sigma} X_{[P} \tilde{D}_{A]}=2 X_{[P} \tilde{D}_{A]}^{\Sigma}$ is conformally invariant and extends the operator $D_{A P}$ as in $[\mathbf{2 9}, \mathbf{3 0}]$ to such 2-dimensional hypersurfaces. It satisfies $D_{A P}^{\Sigma} X_{B}=2 X_{[P} h_{A] B}^{\Sigma}$, where $h_{A B}^{\Sigma}=P_{\Sigma} h_{A B}$ is the intrinsic tractor metric. This is analogous to the usual result $D_{A P} X_{B}=2 X_{[P} h_{A] B}$ and using this and some other elementary identities it is readily verified that the approach to defining the tractor-D as in $[\mathbf{2 9}, \mathbf{3 0}]$ carries over to this case to give the tractor-D operator $D_{A}^{\Sigma}$ as the (clearly conformally invariant) operator on weighted tractor bundles on $\Sigma$ satisfying

$$
h_{\Sigma}^{A B} D_{A(Q}^{\Sigma} D_{|B| P)_{0}}^{\Sigma} f=-X_{(Q} D_{P)_{0}}^{\Sigma} f,
$$

where $(\cdots)_{0}$ denotes trace-free symmetrization over the enclosed indices. Furthermore since this agrees formally with the usual definition and since, as we observed above, $X_{A}$ commutes with $P_{\Sigma}$, one can deduce that (6) and (4) (on $\Sigma$ ) hold in the $n^{\Sigma}=2$ case too. Similarly (3) holds, in that $D_{A}^{\Sigma}$ is given explicitly by $D_{A}^{\Sigma} f=2 w \tilde{D}_{A}^{\Sigma} f-X_{A} \square^{\Sigma} f$, where $\square^{\Sigma}:=\tilde{D}_{B}^{\Sigma} \tilde{D}_{\Sigma}^{B}$.

For the purposes of expanding out the tractor expressions it is useful to observe that, when working within the preferred scales with $H=0$, the tensor $\mathrm{P}_{a b}^{\Sigma}:=\left(\delta_{a}^{a^{\prime}}-N^{a^{\prime}} N_{a}\right)\left(\delta_{b}^{b^{\prime}}-N^{b^{\prime}} N_{b}\right) \mathrm{P}_{a^{\prime} b^{\prime}}$ plays the same role in the tractor formulae on the conformal 2-surface as the usual Rho-tensor $\mathrm{P}_{a b}$ plays in the regular tractor calculus. For example, in such scales, an explicit formula for the tractor connection $\nabla^{\Sigma}$ is again given by (2) if we just replace occurences of $\mathrm{P}_{a b}$ in that formula by $\mathrm{P}_{a b}^{\Sigma}$ (and of course take $\nabla_{a}$ to mean the intrinsic-to- $\Sigma$ Levi-Civita connection and so forth). From another point of view, the conformal transformation properties of $\mathrm{P}_{a b}^{\Sigma}$ ensure that we could build, on $\Sigma$, a canonical principal bundle with a canonical Cartan connection that is closely analogous to the usual Cartan bundle structures on a conformal manifold. (Of course the local structure of this bundle and connection depends on the embedding.) The operator $\nabla^{\Sigma}$ is the corresponding covariant derivative on the induced tractor bundles. 


\section{Normal operators.}

As mentioned above, the operator $\tilde{D}_{A}$ is not conformally invariant when acting on weighted tractors. In fact,

$$
\widehat{\tilde{D}_{A} f}=\tilde{D}_{A} f+X_{A}\left(\Upsilon^{i} \nabla_{i} f+\frac{w}{2} \Upsilon^{i} \Upsilon_{i} f\right),
$$

for $f$ any section of a weighted tractor bundle. On the other hand, since on $\Sigma$ we have $X_{A} N^{A}=0$, it follows immediately that the operator

$$
\delta: \mathcal{E}^{*}[w] \rightarrow \mathcal{E}^{*}[w-1],
$$

given by

$$
\delta f:=N^{A} \tilde{D}_{A} f
$$

is invariant on $\Sigma$. Recall that $\mathcal{E}^{*}[w]$ indicates any tractor bundle of weight $w$. Note that $\delta f=N^{a} \nabla_{a} f-w H f$ where $\nabla_{a}$ is the tractor connection, and so $\delta$ is a Robin operator. The "usual" conformal Robin operator, familiar from the invariant Neumann-type problem associated with $\square$, is recovered by this formula in the special case that $f$ is a scalar density of weight $1-\frac{n}{2}$.

Of course the operator $\delta$ has normal order 1. In the following theorem, we use $\delta$ together with the tractor-D operators to generate higher order analogues $\delta_{\ell}$ of $\delta$. These are used to construct invariant boundary conditions for the interior operator $\square_{k}$, as required for Theorem 8.1 below. The $\delta_{\ell}$ will also be refined, in the proof of Proposition 7.1, to operators $\delta_{\ell}^{\prime}$ which, in tandem with the $\square_{k}$, produce formally self-adjoint boundary problems except when $n$ is even and $k=n$.

Theorem 5.1. Let $k$ be a positive even integer. On a hypersurface $\Sigma$ in a manifold of dimension $n$ and for each positive integer $\ell$ there is a conformally invariant differential operator along $\Sigma$, $\delta_{\ell}$, which maps $\mathcal{E}^{*}\left[\frac{k-n}{2}\right]$ to $\mathcal{E}_{\Sigma}^{*}\left[\frac{k-n-2 \ell}{2}\right]$, given by $\delta_{1}=\delta$, and

$$
\delta_{\ell} \psi= \begin{cases}D_{\Sigma}^{B} \cdots D_{\Sigma}^{A} P_{\Sigma}(\underbrace{D_{A} \cdots D_{B}}_{\ell / 2} \psi) & \text { for } 2 \leq \ell \text { even }, \\ D_{\Sigma}^{B} \cdots D_{\Sigma}^{A} P_{\Sigma}(\underbrace{D_{A} \cdots D_{B}}_{(\ell-1) / 2} \psi) & \text { for } 3 \leq \ell \text { odd },\end{cases}
$$

for $\psi \in \Gamma\left(\mathcal{E}^{*}\left[\frac{k-n}{2}\right]\right)$. If $n$ is odd then the $\delta_{\ell}$ have order and normal order $r=r_{N}=\ell$ for all $\ell \in \mathbb{Z}_{+}$. If $n$ is even then the $\delta_{\ell}$ have order and normal order $r=r_{N}=\ell$ if

$$
\ell+1 \leq k \leq n-2 \quad \text { or } \quad \ell+2 \leq k=n .
$$

For our present purposes, we need only the special cases

$$
\delta_{\ell}: \mathcal{E}\left[\frac{k-n}{2}\right] \rightarrow \mathcal{E}_{\Sigma}\left[\frac{k-n-2 \ell}{2}\right],
$$


(i.e., no tractor indices). However these hypersurface differential operators are strongly invariant in the sense discussed for differential operators on tensor densities on $M$ in [21]. That is, they remain invariant operators upon tensoring of the domain and target bundles with any tractor bundle. It is just required that in the construction, as for densities here, one replaces, where required, the Levi-Civita connection with the appropriate coupled Levi-Civita-tractor connection.

Proof of the theorem. The conformal invariance of the displayed operators in the theorem is clear, since the ingredients $D_{A}, \delta, P_{\Sigma}$ and $D_{\Sigma}^{A}$ are all conformally invariant operators (though $P_{\Sigma}$ has order 0 ). The weight of the target bundle is correct since each of $D_{A}, \delta$, and $D_{\Sigma}^{A}$ lowers the weight by 1 unit while $P_{\Sigma}$ leaves the weight unchanged. From these weights one can easily deduce that the order of the operators is $\leq \ell$. It is also clear that these operators are independent of how the normal $N^{a}$ (used in the construction of $\delta$ and $P_{\Sigma}$ ) is extended off the hypersurface, since the $D_{\Sigma}^{A}$ involve only differentiation in directions tangent to the hypersurface. Thus it remains to establish that the operators have the normal order claimed.

Let us consider first $\ell$ odd, and $\ell$ and $k$ as in the last part of the theorem. (That is if $n$ is odd then $\ell \in \mathbb{Z}_{+}$. If $n$ is even then $\ell+1 \leq k \leq n-2$ or $\ell+2 \leq k=n$.) Using (3), one quickly establishes that the operator

$$
\delta \underbrace{D_{A} \cdots D_{B}}_{(\ell-1) / 2} \psi
$$

has principal part

$$
X_{A} \cdots X_{B} \delta \square^{(\ell-1) / 2} \psi .
$$

That is, term (8) has order $r=\ell$, and term (7) minus term (8) has order at most $\ell-1$. At leading order, the operator $\delta \square^{(\ell-1) / 2} \psi$ agrees with $N^{a} \nabla_{a} \Delta^{(\ell-1) / 2} \psi$. It is easily verified that the operator $N^{a} \nabla_{a} \Delta^{(\ell-1) / 2}$ has normal order $r_{N}=\ell$. Since, on $\Sigma, P_{\Sigma}\left(X_{A}\right)=X_{A}$, it follows that (8) survives in

$$
P_{\Sigma}(\delta \underbrace{D_{A} \cdots D_{B}}_{(\ell-1) / 2} \psi)
$$

so that

$$
P_{\Sigma}(\delta \underbrace{D_{A} \cdots D_{B}}_{(\ell-1) / 2} \psi)-X_{A} \cdots X_{B} \delta \square^{(\ell-1) / 2} \psi
$$

also has order at most $\ell-1$. The operator $D_{\Sigma}^{A}$, being intrinsic to the hypersurface $\Sigma$, has normal order 0 . Thus the normal order of

$$
D_{\Sigma}^{B} \cdots D_{\Sigma}^{A}\left[P_{\Sigma}\left(\delta D_{A} \cdots D_{B} \psi\right)-X_{A} \cdots X_{B} \delta \square^{(\ell-1) / 2} \psi\right]
$$


is at most $\ell-1$. Now it follows from (6) that

$$
D_{\Sigma}^{B} \cdots D_{\Sigma}^{A}\left(X_{A} \cdots X_{B} \delta \square^{(\ell-1) / 2} \psi\right)
$$

is a multiple of $\delta \square^{(\ell-1) / 2} \psi$, and so has normal order $r_{N}=\ell$ if it is not zero. Combining this with the immediately previous observation, we have that $D_{\Sigma}^{B} \cdots D_{\Sigma}^{A} P_{\Sigma}\left(\delta D_{A} \cdots D_{B} \psi\right)$ also has normal order $\ell$ provided $D_{\Sigma}^{B} \cdots D_{\Sigma}^{A}\left(X_{A}\right.$ $\left.\cdots X_{B} \delta \square^{(\ell-1) / 2} \psi\right) \neq 0$. In fact $\delta \square^{(\ell-1) / 2} \psi$ has weight $\frac{k-n-2 \ell}{2}$, so

$$
\begin{aligned}
& D_{\Sigma}^{B} \cdots D_{\Sigma}^{A}\left(X_{A} \cdots X_{B} \delta \square^{(\ell-1) / 2} \psi\right) \\
& =\left(\prod_{i=1}^{(\ell-1) / 2} \frac{1}{2}(k+2 i-2 \ell-1)(n+k+2 i-2 \ell-4)\right) \delta \square^{(\ell-1) / 2} \psi,
\end{aligned}
$$

which is a non-zero multiple of $\delta \square^{(\ell-1) / 2} \psi$ as required. (To see this note that the factors $(k+2 i-2 \ell-1)$ are odd integers. Similarly if $n$ is odd then the factors $(n+k+2 i-2 \ell-4)$ are odd numbers. On the other hand if $n$ is even then the factors $(n+k+2 i-2 \ell-4)$ are positive since $n+k-2 \ell-2>0$ for $k$ and $\ell$ as in the theorem.)

The proof for $\ell$ even is almost identical. In this case, by an analogue of the arguments above one quickly deduces that the operators have the normal order claimed in the last part of the theorem if, in general,

$$
D_{\Sigma}^{B} \cdots D_{\Sigma}^{A}(\underbrace{X_{A} \cdots X_{B}}_{\ell / 2} \square^{\ell / 2} \psi) \neq 0
$$

for $\psi \in \Gamma\left(\mathcal{E}^{*}\left[\frac{k-n}{2}\right]\right)$. In this case we get

$$
\begin{aligned}
& D_{\Sigma}^{B} \cdots D_{\Sigma}^{A}\left(X_{A} \cdots X_{B} \square^{\ell / 2} \psi\right) \\
& =\left(\prod_{i=1}^{\ell / 2} \frac{1}{2}(k+2 i-2 \ell-1)(n+k+2 i-2 \ell-4)\right) \square^{\ell / 2} \psi
\end{aligned}
$$

(cf. the case of $\ell$ odd). This does not vanish for $k$ and $\ell$ as in the last part of the theorem for precisely the same reasons as for the case of $\ell$ odd.

We now specialize to the case of conformal manifolds of positive definite metric signature. We would like to use Theorem 5.1 to construct conformally invariant odd-order pseudo-differential operators of boundary type, via a process analogous to the construction of the conformal Dirichlet-toNeumann operator. The idea is that the family of operators $\delta_{\ell}$ should provide conformally invariant boundary conditions for the $\square_{k}$. The resulting boundary problems are not a priori formally self-adjoint, but in most cases admit formally self-adjoint modifications, obtained by replacing the $\delta_{\ell}$ by operators $\delta_{\ell}^{\prime}$ whose construction is motivated by integration by parts formulas. 
In the next section, we digress briefly to review some analytic considerations relevant to the construction of these pseudo-differential operators.

\section{Boundary problems.}

Let $M$ be a Riemannian manifold with boundary $\Sigma$. Let $k$ be a positive even integer. There are some natural boundary problems, based on the interior operator $(-\Delta)^{k / 2}$, which admit an elementary description, and which are quite well-behaved with respect to elliptic theory. Let $N$ be the inward unit normal vector field, and let

$$
S_{m} u= \begin{cases}\Delta^{m / 2} u, & m=0,2, \ldots, k-2, \\ \Delta^{(m-1) / 2} \nabla_{N} u & m=1,3, \ldots, k-1,\end{cases}
$$

where $\Delta^{0}$ is understood as 1 . Let $\mathbf{m}=\left(m_{j}\right)_{j=1}^{k / 2}$ be a list of $k / 2$ integers from the range $0,1, \ldots, k-1$, with $m_{1}<\ldots<m_{k / 2}$. For notational convenience, we shall view $\mathbf{m}$ both as a set (so that, for example, $m \in \mathbf{m}$ makes sense), and as an ordered $k / 2$-tuple. Let

$$
S_{\mathbf{m}}=\left(S_{m_{1}}, \ldots, S_{m_{j}}\right) .
$$

Then the pair (or problem) $\left((-\Delta)^{k / 2}, S_{\mathbf{m}}\right)$ satisfies several ellipticity properties. We digress for a moment to describe these properties. Some general references are $[37,36,27,32]$.

Let $(M, \Sigma)$ be a compact Riemannian manifold with boundary, and let $A$ be a scalar differential operator of order $k$ on $M$. For purposes of discussing boundary problems, $A$ is said to be elliptic if its leading symbol $a_{k}$ satisfies

$$
\left|a_{k}(x, \xi)\right| \geq c_{0}|\xi|^{k} \text { on } M,
$$

for some constant $c_{0}>0$, where $|\xi|^{2}=g^{a b} \xi_{a} \xi_{b}$. A is strongly elliptic if

$$
\operatorname{Re} a_{k}(x, \xi) \geq c_{1}|\xi|^{k} \text { on } M,
$$

for some constant $c_{1}>0$.

If $n \geq 3$, any elliptic differential operator has even order ([36], Section 6.1). Thus we shall assume from now on that $k$ is even. $A$ is properly elliptic if for all $x \in \Sigma$ and $\eta_{a} \in \mathcal{E}_{a}$ not parallel to the inward unit normal cotangent vector $N_{a}$ at $x$, the roots of the polynomial equation

$$
a_{k}\left(x, \eta_{a}+\tau N_{a}\right)=0
$$

( $\tau$ being the indeterminate) are separated by the real axis; that is, the roots can be labelled

$$
\tau_{i}^{ \pm}, \quad \text { where } \pm \operatorname{Im} \tau_{i}^{ \pm}>0
$$

for $i=1, \ldots, k / 2$. 
Note that operators with principal part $(-\Delta)^{k / 2}$ are elliptic, strongly elliptic, and properly elliptic. Indeed,

$$
g^{a b}\left(\eta_{a}+\tau N_{a}\right)\left(\eta_{b}+\tau N_{b}\right)=\tau^{2}+2 b \tau+c^{2},
$$

where

$$
b=\eta_{a} N^{a}, \quad c^{2}=g^{a b} \eta_{a} \eta_{b} .
$$

Since $\eta_{a}$ is not parallel to $N_{a}$, we have $|b|<c$ by the Schwarz inequality. Thus for each $j$, we have $\tau_{j}^{ \pm}=-b \pm \sqrt{-1} \beta$, where $\beta=\sqrt{c^{2}-b^{2}}$. (In fact, strong ellipticity and even order imply proper ellipticity [37].)

The basic well-posedness condition for boundary problems is the so-called Lopatinski-Shapiro condition. This can be stated in terms of an ordinary differential equations problem, or equivalently, as an algebraic problem (which arises in the study of this ODE problem). We shall state the algebraic form. Let $\left(B_{m_{j}}\right)$ be a list of $k / 2$ scalar operators, defined near the boundary, of orders $0 \leq m_{1}<\ldots<m_{k / 2}<k$. Let $b_{m_{j}}$ be the leading symbol of $B_{m_{j}}$. Let

$$
M^{ \pm}=\prod_{i=1}^{k / 2}\left(\tau-\tau_{i}^{ \pm}\right) .
$$

Consider the polynomials $b_{m_{j}}\left(x, \eta+\tau N_{x}\right)$. The Lopatinski-Shapiro (henceforth $L S$ ) condition is the requirement that these polynomials are linearly independent in $\mathbb{C}[\tau]$ modulo the principal ideal generated by $M^{+}$. (As before, $\eta$ is taken not parallel to $N_{x}$.)

A system of boundary operators $B_{\mathbf{m}}$ is normal if $b_{m_{j}}\left(x, N_{x}\right) \neq 0$ for all $j=1, \ldots, k / 2$. In the normal case, we may express

$$
B_{m_{j}}=\sum_{q=0}^{m_{j}} B_{m_{j}, q}\left(\nabla_{N}\right)^{q},
$$

where $B_{m_{j}, q}$ is a tangential operator of order at most $m_{j}-q$. In particular, $B_{m_{j}, m_{j}}$ is a function, and

$$
b_{m_{j}}\left(x, N_{x}\right)=B_{m_{j}, m_{j}}(x) .
$$

Normality is thus just the condition that the functions $B_{j, m_{j}}$ are nowhere vanishing.

Note that ellipticity, strong ellipticity, and proper ellipticity are properties of the operator $A$ alone, while normality is a property of the system of boundary operators alone. The LS condition, in contrast, is a joint property of $A$ and the boundary operators. All properties are insensitive to changes in the operators $A$ and $B_{m_{j}}$ which preserve the principal part. They are also insensitive to multiplication of $A$ by a positive constant, or multiplication of any $B_{m_{j}}$ by a non-zero constant. 
Remark 6.1. For the problems $\left((-\Delta)^{k / 2}, S_{\mathbf{m}}\right)$, the LS condition amounts to checking that the polynomials

$$
\begin{cases}((\tau-\alpha)(\tau-\bar{\alpha}))^{m_{j} / 2}, & \left(m_{j} \text { even }\right), \\ (\tau+b)((\tau-\alpha)(\tau-\bar{\alpha}))^{\left(m_{j}-1\right) / 2}, & \left(m_{j} \text { odd }\right),\end{cases}
$$

where $\alpha=-b+\sqrt{-1} \beta$, are linearly independent modulo $(\tau-\alpha)^{k / 2}$; this is straightforward. Normality for the $S_{\mathbf{m}}$ is also clear, as each $S_{m_{j}, m_{j}}$ is \pm 1 or $\pm \sqrt{-1}$.

The pair $\left((-\Delta)^{k / 2}, S_{\mathbf{m}_{\mathrm{D}}}\right)$, where

$$
\mathbf{m}=\mathbf{m}_{\mathrm{D}}:=(0,2, \ldots, k-2)
$$

is called the iterated Dirichlet problem for $(-\Delta)^{k / 2}$. This is intuitively related to its $k=2$ special case, the Dirichlet problem for $-\Delta$, as follows. A solution $u$ of

$$
\left\{\begin{array}{l}
\Delta^{k / 2} u=0, \\
\left.\left(\Delta^{q / 2} u\right)\right|_{\Sigma}=f_{q}, \quad q=0,2, \ldots, k-2,
\end{array}\right.
$$

may be identified with a solution $\left(u_{0}, u_{2}, \ldots, u_{k-2}\right)$ of

$$
\left\{\begin{array}{l}
\Delta u_{q}=u_{q+2} \\
\left.u_{q}\right|_{\Sigma}=f_{q}
\end{array}\right.
$$

where we take $u_{k}$ to be 0 . The idea is to solve successively for $u_{k-2}, u_{k-4}$, $\ldots, u_{0}$. Similar remarks hold for the iterated Neumann problem, which comes from choosing

$$
\mathbf{m}=\mathbf{m}_{\mathrm{N}}:=(1,3, \ldots, k-1) .
$$

Another standard problem is the generalized Dirichlet problem, which comes from taking

$$
\mathbf{m}=\mathbf{m}_{0}:=(0,1, \ldots, k / 2-1) .
$$

Remark 6.2. The boundary problems in which we are interested have principal parts which are closely related to those of the problems $\left((-\Delta)^{k / 2}, S_{\mathbf{m}}\right)$. After a choice of scale, the interior operator $\square_{k}$ has the form $\Delta^{k / 2}+$ (lower order). In fact, by Riemannian invariant theory, "lower order" in this formula is actually order at most $k-2$ : The difference $\square_{k}-\Delta^{k / 2}$ is a term coupling the Riemann curvature to a derivative of order $k-2$, plus lower-order terms. Some further invariant theory, which also takes account of invariants of the boundary imbedding, is involved in the study of the boundary operators. The boundary operators $\delta_{\ell}$ we have constructed (as well as their modifications $\delta_{\ell}^{\prime}$ which we shall construct below) have coefficients which are polynomial in the jets of the metric and its inverse, and in 
the jets of the defining function of $\Sigma$. Any operators so constructed, and which are of normal order $q$ and constructed to scale according to a rule

$$
B\left(\alpha^{2} g\right)=\alpha^{-q} B(g)
$$

under uniform dilation $g \mapsto \alpha^{2} g, 0<\alpha \in \mathbb{R}$ of the metric $g$, must have the form

$$
c_{q}\left(\nabla_{N}\right)^{q}+\sum_{m=0}^{q-1} D_{m}\left(\nabla_{N}\right)^{m}
$$

where $c_{q}$ is a non-zero constant, and the $D_{m}$ are differential operators on $\Sigma$ (i.e., tangential differential operators) of order at most $q-m$. Operators that arise from conformally invariant constructions are naturally endowed with a homogeneity condition of the form (12) in each chosen scale $g$, and $q$ is in fact the difference between weights of the source and target line bundles. One uses these homogeneity properties, just as for "ordinary" $\mathrm{O}(n)$ invariant theory, to count derivatives. Another application of invariant theory shows that the principal part of the operator (13) must have the form

$$
\begin{cases}c_{q}\left(\nabla_{N}\right)^{q}+c_{q-2} \Delta_{\Sigma}\left(\nabla_{N}\right)^{q-2}+\ldots+c_{0} \Delta_{\Sigma}^{q / 2} & (q \text { even }), \\ c_{q}\left(\nabla_{N}\right)^{q}+c_{q-2} \Delta_{\Sigma}\left(\nabla_{N}\right)^{q-2}+\ldots+c_{1} \Delta_{\Sigma}^{(q-1) / 2} \nabla_{N} & (q \text { odd }),\end{cases}
$$

where the $c_{i}$ are constants.

The following result will be useful.

Lemma 6.3. Let $A$ be a properly elliptic scalar differential operator of (positive even) order $k$ on $M$, and let $B_{\mathbf{m}}$ and $B_{\mathbf{m}}^{\prime}$ be systems of boundary operators, both with the same list of orders $\mathbf{m}: 0 \leq m_{1}<\ldots<m_{k / 2}<k$, and with

$$
B_{m_{j}}^{\prime}=c_{m_{j}} B_{m_{j}}+\sum_{i<j} D_{j, i} B_{m_{i}}, \quad j=1, \ldots, k / 2,
$$

where the $D_{j, i}$ are tangential differential operators of orders $\leq m_{j}-m_{i}$, and the $c_{m_{j}}$ are nowhere-vanishing functions. Suppose that $B_{\mathbf{m}}$ satisfies the LS condition relative to $A$. Then $B_{\mathbf{m}}^{\prime}$ also satisfies the LS condition relative to A.

Proof. The leading symbol $b_{m_{j}}^{\prime}$ of $B_{m_{j}}^{\prime}$, evaluated at $\left(x, \eta+\tau N_{x}\right)$, is

$$
c_{m_{j}}(x) b_{m_{j}}\left(x, \eta+\tau N_{x}\right)+\sum_{i<j} d_{j, i}(x, \eta) b_{m_{i}}\left(x, \eta+\tau N_{x}\right),
$$

where $d_{j, i}$ is the order $m_{j}-m_{i}$ symbol of $D_{j, i}$. Thus for a given $x$, the list of cosets

$$
b_{m_{j}}^{\prime}\left(x, \eta+\tau N_{x}\right)+\mathbb{C}[\tau] M^{+}(\tau)
$$


(arranged, say, as a column vector) is an invertible lower triangular complex matrix times the list of cosets

$$
b_{m_{j}}\left(x, \eta+\tau N_{x}\right)+\mathbb{C}[\tau] M^{+}(\tau) .
$$

Let $(A, B)$ be a properly elliptic, normal boundary problem satisfying the LS condition. If $(A, B)$ is in addition formally self-adjoint (see below), it admits, on a compact manifold, a discrete spectral resolution $\left(\phi_{j}, \lambda_{j}\right)$, in which the eigenvalues $\lambda_{j}$ are real.

The $\phi_{j}$ are a complete orthonormal set in $L^{2}(M)$, and satisfy $\left.\left(B \phi_{j}\right)\right|_{\Sigma}=0$. This gives a realization of the boundary problem $(A, B)$ as an unbounded operator in $L^{2}$,

$$
A_{B}: \sum c_{j} \phi_{j} \mapsto \sum c_{j} \lambda_{j} \phi_{j}
$$

On the other hand, if $\underline{B} u:=\left.(B u)\right|_{\Sigma}$, we may consider the operator $(A, \underline{B})$.

For a properly elliptic problem that satisfies the LS condition and is normal, the index (dim ker minus codim range) of $(A, \underline{B})$ agrees with that of $A_{B}$ ([32], Section 4.3). A key point is that normality guarantees the surjectivity of $\underline{B}$. The advantage of having a formally self-adjoint problem is that, owing to (15), the index must vanish. As a result, the two deficiency indices (dim ker and codim range) are equal, and thus the vanishing of one is equivalent to the vanishing of the other. This means that we can guarantee unique solvability of a formally self-adjoint problem by making an elementary assumption of vanishing kernel. Such an assumption is "elementary" because, by elliptic regularity, a function in the kernel is smooth; thus $\operatorname{ker} A_{B}=\operatorname{ker}(A, \underline{B})$, and vanishing kernel is a condition that is independent of choices of function spaces. As a result, for the problem

$$
A u=0, \underline{B} u=\beta
$$

(with, say, $\beta$ smooth), we have the following alternative: (1) $\operatorname{ker} A_{B}=0$, and solutions to (16) always exist and are unique; or (2) ker $A_{B} \neq 0$, and (16) has no solution for some $\beta$, and a non-unique solution for some $\beta^{\prime}$. An example of the second situation is the Neumann problem for the Laplacian. The function 1 has vanishing Neumann data, and is annihilated by the Laplacian. As a result, in view of (15), there is no $L^{2}$ function (thus no smooth function) with vanishing Neumann data such that $\Delta u=1$.

We sum up the properties we shall be using in the following proposition; see [34], Section 20.1 and [32], Section 4.3 for proofs.

Proposition 6.4. Let $(A, B)$ be a properly elliptic, normal boundary problem satisfying the LS condition on a manifold with boundary $(M, \Sigma)$. Then the kernel of $(A, \underline{B})$ is finite-dimensional, and consists of smooth functions. If in addition $(A, B)$ is formally self-adjoint, then 
(a) the codimension of the range of the realization $A_{B}$ of $(A, B)$ in $L^{2}(M)$ equals the dimension of the kernel of $(A, \underline{B})$, and $\operatorname{ker}\left(A_{B}\right)=\operatorname{ker}(A, \underline{B})$;

(b) whenever $\operatorname{ker}(A, \underline{B})=0$, the problem $A u=0$ with prescribed $\underline{B} u$ is uniquely solvable, and there is a well-defined solution operator carrying smooth data $\underline{B} u$ to smooth $u$.

Motivated by this, we shall now investigate formal self-adjointness properties for our conformally invariant boundary problems.

\section{Formal self-adjointness.}

A boundary problem $(A, B)$ is formally self-adjoint iff

$$
\int_{M}[(A u) v-u A v]=0
$$

whenever $B u=B v=0$ on $\Sigma$. A necessary condition for formal selfadjointness is that the interior operator $A$ be formally self adjoint. Any such $A$ satisfies

$$
\int_{M}[(A u) v-u A v]=-\int_{\Sigma} \operatorname{sk}_{A}(u, v)
$$

where $\operatorname{sk}(u, v)$ is a skew bilinear form on the Cauchy data and its tangential derivatives at the boundary $\Sigma$. Clearly, at each point, $\operatorname{sk}_{A}(u, v)$ has maximal isotropic subspaces of at least half the fiber dimension of the Cauchy data bundle. Given such an operator $A$, which is formally self-adjoint on the interior, if one can demonstrate that the kernel of the boundary operator $B$ lies inside such an isotropic subspace at each point of the boundary, then the problem $(A, B)$ is formally self-adjoint.

A good example of this is given by the usual powers of the Laplacian $\Delta^{k / 2}$ on Riemannian manifolds. So, for the moment, suppose we have fixed a choice of scale so that $M$ is a Riemannian manifold with Riemannian boundary manifold $\Sigma$. Then one easily obtains that

$$
\int_{M}\left(u \Delta^{k / 2} v-v \Delta^{k / 2} u\right)=-\int_{\Sigma} \operatorname{sk}_{\Delta^{k / 2}}(u, v),
$$

where

$$
\operatorname{sk}_{\Delta^{k / 2}}(u, v)=\sum_{i=0}^{k / 2-1}\left[\left(\Delta^{i} u\right) \nabla_{N} \Delta^{k / 2-1-i} v-\left(\Delta^{i} v\right) \nabla_{N} \Delta^{k / 2-1-i} u\right] .
$$

Thus the operator is formally self-adjoint with for any of the standard choices of boundary operators $S_{\mathbf{m}}$ from the beginning of Section 6 with the property that

$$
\left\{m_{j} \mid 1 \leq j \leq k / 2\right\} \cap\left\{k-1-m_{j} \mid 1 \leq j \leq k / 2\right\}=\emptyset .
$$


In particular, we have formal self-adjointness for the iterated Dirichlet problem (9), the iterated Neumann problem (10), and the generalized Dirichlet problem (11).

Returning to the conformal setting, the following proposition asserts that there exist modifications $\delta_{i}^{\prime}$ of the boundary operators $\delta_{i}$ so that analogous results on self-adjointness hold for the operators $\square_{k}$. The proposition does not cover the case $k=n$ even.

Proposition 7.1. For $n$ even let $k \in\{0,2, \ldots, n-2\}$ and for $n$ odd let $k \in 2 \mathbb{Z}_{+}$. For each such $k$ and each $\mathbf{m}$ satisfying (17), there exist conformally invariant normal boundary operators $\delta_{\mathbf{m}}^{\prime}$ such that $\left(\square_{k}, \delta_{\mathbf{m}}^{\prime}\right)$ is formally selfadjoint. In particular, this is the case for $\mathbf{m}=\mathbf{m}_{\mathrm{D}}=(0,2, \ldots, k-2)$, $\mathbf{m}=\mathbf{m}_{\mathrm{N}}=(1,3, \ldots, k-1)$, and $\mathbf{m}=\mathbf{m}_{0}=(0,1, \ldots, k / 2-1)$.

The last sentence of the proposition indicates that there will be natural formally self-adjoint problems of iterated Dirichlet, iterated Neumann, and generalized Dirichlet type.

Before we treat the proposition some preliminary observations are useful. First we note that the tractor construction of the operators $\square_{k}$ produces operators which are formally self-adjoint on the interior. This observation was first made by Eastwood [22]. In fact, we could replace any choice of an invariant $\square_{k}$ by the formally self-adjoint operator $\left(\square_{k}+\square_{k}^{*}\right) / 2$ without disturbing invariance. This is because (1) a conformally invariant operator $D: \mathcal{E}\left[-\frac{n}{2}+r\right] \rightarrow \mathcal{E}\left[-\frac{n}{2}+s\right]$ has $D^{*}: \mathcal{E}\left[-\frac{n}{2}-s\right] \rightarrow \mathcal{E}\left[-\frac{n}{2}-r\right]$ conformally invariant; and (2) the weights of $\square_{k}$ are symmetric about $-n / 2$. The observation that the tractor construction of $\square_{k}$ is formally self-adjoint, however, will be valuable to us below: To construct boundary operators that lead to formally self-adjoint boundary problems, we need at least some explicit knowledge of the formally self-adjoint interior operator we are using. This is provided by the explicit formula in Proposition 4.1.

Since the coupled tractor-Levi-Civita connection $\nabla_{a}$ satisfies a Leibniz rule over sections of tractor bundles and tensor bundles, it follows that it satisfies an obvious generalization of the usual "integration by parts" formula. For example, if $\psi^{a B} \in \Gamma \mathcal{E}^{a B}[w]$ and $\phi_{B} \in \Gamma \mathcal{E}_{B}[-n-w]$ then, since we take $N^{a}$ to be an inward normal, we have

$$
\int_{M} \phi_{B} \nabla_{a} \psi^{a B}=-\int_{\Sigma} \phi_{B} N_{a} \psi^{a B}-\int_{M}\left(\nabla_{a} \phi_{B}\right) \psi^{a B}
$$

and this remains true even if instead $\phi$ and $\psi$ have further tractor indices which are contracted together. Using the Leibniz property of the tractorLevi-Civita connection one may also easily show that if $\psi^{A} \in \Gamma \mathcal{E}^{A}[w]$ and $\phi \in \Gamma \mathcal{E}[1-n-w]$, then

$$
\phi D_{A} \psi^{A}=\left(D_{A} \phi\right) \psi^{A}+\nabla_{a} V^{a}
$$


where, if $\left[\psi_{A}\right]_{g}=\left(\rho \psi_{a} \sigma\right), V^{a}$ is the conformally invariant bilinear operator given by $\left(\phi, \psi^{A}\right) \mapsto \sigma \nabla^{a} \phi-\phi \nabla^{a} \sigma+(n+2 w) \phi \psi^{a}$. (Note that (18) and (19) hold on $\Sigma$ regarded as an intrinsic conformal manifold, except that in the $n^{\Sigma}=2$ case one must use the special tractor calculus described in Section 4.) This yields the following integration formula:

$$
\begin{aligned}
\int_{M} \phi D_{A} \psi^{A}=\int_{M} & \left(D_{A} \phi\right) \psi^{A} \\
& -\int_{\Sigma}\left[(n+2 w) \phi N_{A} \psi^{A}-\phi \delta X_{A} \psi^{A}+(\delta \phi) X_{A} \psi^{A}\right] .
\end{aligned}
$$

Furthermore this equation also is valid even if $\phi$ and $\psi^{A}$ have other tractor indices which are contracted together. Combining these results, it is a trivial exercise to verify that each operator

$$
\square_{k}:=D^{A} \cdots D^{B} \square \underbrace{D_{B} \cdots D_{A}}_{(k-2) / 2}
$$

is self-adjoint on the interior.

In fact more follows if we are just a little more observant. Notice that the term $\phi_{B} N_{a} \psi^{a B}$ in (18) is conformally invariant. Similarly notice the conformal invariance of each term in the integrands of the integrals on the right hand side of (20). It follows immediately that for the operators $\square_{k}$, (with $k$ as in the proposition) one can produce a pointwise skew form $\operatorname{sk}_{\square_{k}}^{\prime}$ which is conformally invariant and satisfies $\int_{M}\left(u \square_{k} v-v \square_{k} u\right)=$ $-\int_{\Sigma} \operatorname{sk}_{\square_{k}}^{\prime}(u, v)$. (Of course it was already clear that the global skew form $(u, v) \mapsto \int_{\Sigma} \operatorname{sk}_{\square_{k}}^{\prime}(u, v)$ is conformally invariant.)

Let us now turn our attention to the example $\square_{4}$. Treating this case illustrates an easy case of the general procedure we use to establish the proposition. It is easily shown (see e.g., [30]) that if $v \in \Gamma \mathcal{E}\left[2-\frac{n}{2}\right]$ then $2(4-n) \square D_{A} v=X_{A} \square_{4} v$. It follows immediately that for $u, v \in \Gamma \mathcal{E}\left[2-\frac{n}{2}\right]$ one has $u D^{A} \square D_{A} v=\left(D^{A} u\right) \square D_{A} v$ and, in particular

$$
\int_{M} u D^{A} \square D_{A} v=\int_{M}\left(D^{A} u\right) \square D_{A} v .
$$

Thus

$$
\int_{M}\left(u \square_{4} v-v \square_{4} u\right)=-\int_{\Sigma} \operatorname{sk}_{\square_{4}}^{\prime}(u, v)
$$

where

$$
\begin{aligned}
\operatorname{sk}_{\square_{4}}^{\prime}(u, v) & =\left(D^{B} u\right) N^{a} \nabla_{a} D_{B} v-\left(D^{B} v\right) N^{a} \nabla_{a} D_{B} u \\
& =\left(D^{B} u\right) \delta D_{B} v-\left(D^{B} v\right) \delta D_{B} u
\end{aligned}
$$


Note that the last line expresses $\mathrm{sk}_{\square_{4}}^{\prime}$ as the difference of two conformally invariant bilinear operators. Of course (21) really only determines this bilinear operator up to the addition of $\nabla^{\Sigma}$ divergences and in fact it is prudent to choose a representative that is more closely analogous to $\mathrm{sk}_{\Delta^{2}}$. In this case it is rather elementary to find such a representative. First observe that for $u \in \Gamma \mathcal{E}\left[2-\frac{n}{2}\right]$ one has

$$
D^{B} u=2\left(D_{\Sigma}^{B} u+N^{B} \delta u+(2-n)^{-1} X^{B} \delta_{2} u\right) .
$$

So by (20), applied to the $D_{A}^{\Sigma}$ of the boundary manifold, one has

$$
\begin{aligned}
\int_{\Sigma}\left(D^{B} u\right) \delta D_{B} v= & 2 \int_{\Sigma} u D_{\Sigma}^{B} P_{\Sigma} \delta D_{B} v+(\delta u) N^{B} \delta D_{B} v \\
& +(2-n)^{-1}\left(\delta_{2} u\right) X^{B} \delta D_{B} v \\
= & 2 \int_{\Sigma}\left(\delta_{0} u\right) \delta_{3} v+\left(\delta_{1} u\right) N^{B} \delta_{1} D_{B} v-\left(\delta_{2} u\right) \delta_{1} v
\end{aligned}
$$

where, in the last line, we have written $\delta_{0} u$ for $\left.u\right|_{\Sigma}$ and $\delta_{1}$ for $\delta$. Thus we have

$$
\int_{\Sigma} \operatorname{sk}_{\square_{4}}^{\prime}(u, v)=\int_{\Sigma} \operatorname{sk}_{\square_{4}}(u, v),
$$

where

$$
\begin{aligned}
& \operatorname{sk}_{\square_{4}}(u, v)=2\left[\left(\delta_{0} u\right) \delta_{3} v+(\right.\left.\delta_{1} u\right)\left(N^{B} \delta_{1} D_{B} v+\delta_{2} v\right) \\
&\left.-\left(\delta_{1} v\right)\left(N^{B} \delta_{1} D_{B} u+\delta_{2} u\right)-\left(\delta_{0} v\right) \delta_{3} u\right] .
\end{aligned}
$$

This immediately suggests taking $\delta_{\mathbf{m}_{\mathrm{D}}}^{\prime}=\left(\delta_{0}^{\prime}, \delta_{2}^{\prime}\right), \delta_{\mathbf{m}_{\mathrm{N}}}^{\prime}=\left(\delta_{1}^{\prime}, \delta_{3}^{\prime}\right)$, or $\delta_{\mathbf{m}_{0}}^{\prime}=$ $\left(\delta_{0}^{\prime}, \delta_{1}^{\prime}\right)$, where

$$
\delta_{0}^{\prime}:=\delta_{0}, \delta_{1}^{\prime}:=\delta_{1}, \delta_{2}^{\prime}:=\left(\delta_{2}+N^{B} \delta_{1} D_{B}\right), \delta_{3}^{\prime}:=\delta_{3},
$$

since then it is immediately clear that $\left(\square_{4}, \delta_{\mathbf{m}_{\mathrm{D} \text { or or } 0}}^{\prime}\right)$ is formally selfadjoint. In fact these problems are good choices, since they are clearly conformally invariant, they are well-defined without choosing an extension of $N^{a}$ (or $N^{A}$ ) off $\Sigma$, and it is easily verified explicitly that (given now some arbitrary choice of extension of $N^{a}$ off $\Sigma$ ) the $\delta_{i}^{\prime}$ are of the form

$$
\begin{aligned}
\delta_{1}^{\prime} u & =\nabla_{N} u+\text { lower normal order terms, } \\
\delta_{2}^{\prime} u & =-\frac{n-4}{2}\left(\nabla_{N}\right)^{2} u+\text { lower normal order terms, } \\
\delta_{3}^{\prime} u & =\frac{n-4}{2}\left(\nabla_{N}\right)^{3} u+\text { lower normal order terms. }
\end{aligned}
$$

In particular this form implies that (for $n \neq 4$ ) the leading-normal-order part of $\mathrm{sk}_{\square_{4}}$ recovers the leading-normal-order part of $\mathrm{sk}_{\Delta^{2}}$ up to an overall factor. In fact there is some choice in constructing the boundary operators. Note for example that in the right hand side of (22) we could have first reexpressed $N^{A} \delta D_{A}$ in terms of $\delta_{2}, \delta_{1}, \delta_{0}$ and their $\nabla^{\Sigma}$ derivatives. Using this 
observation and rearranging the result appropriately can lead to a different set of conformally invariant boundary operators.

The alert reader will have noticed that we have not mentioned the boundary operator list complementary to $\delta_{\mathbf{m}_{0}}^{\prime}$, that being $\left(\delta_{2}^{\prime}, \delta_{3}^{\prime}\right)$. One reason is that for the Riemannian problem which this generalizes, $\Delta^{2}$ with $\left(\left(\nabla_{N}\right)^{2},\left(\nabla_{N}\right)^{3}\right)$, the LS condition is not satisfied. In fact, it is easily seen that for the Riemannian problem $(-\Delta)^{k}$ with $\left(\left(\nabla_{N}\right)^{m}\right)_{m \in \mathbf{m}}$, a necessary condition for LS is that $\{0,1\} \cap \mathbf{m} \neq \emptyset$.

In the proof of Proposition 7.1 below we describe one 'once and for all' choice of such boundary operators (which, for $\square_{4}$ and the boundary operator $B_{\mathrm{D} \text { or N or 0 }}$, agrees with the choice above). Before we describe this proof we need one more preliminary result.

Lemma 7.2. Let $\mathcal{V}$ be a vector bundle on the boundary manifold $\Sigma$. With $k$ as in the proposition, any conformally invariant linear differential operator

$$
I: \mathcal{E}\left[\frac{k-n}{2}\right] \rightarrow \mathcal{V}
$$

of order $r$ and normal order $r_{N} \leq k-1$ determines a canonical bundle homomorphism

$$
H(I): \oplus_{i=0}^{r_{N}} \mathcal{E}_{\mathcal{E}^{\perp \cdots B}}^{\Sigma}\left[\frac{k-n-2 r}{2}\right] \rightarrow \mathcal{V}
$$

and the operator I is given as composition of this with the differential operator

$$
\mathbf{D}: \mathcal{E}\left[\frac{k-n}{2}\right] \rightarrow \oplus_{i=0}^{r_{N}} \mathcal{E}_{\mathcal{E}^{\perp \cdots B}}^{\Sigma}\left[\frac{k-n-2 r}{2}\right]
$$

given by

$$
f \mapsto(\underbrace{D_{A}^{\Sigma} \cdots D_{G}^{\Sigma}}_{r} \delta_{0} f, \underbrace{D_{A}^{\Sigma} \cdots D_{F}^{\Sigma}}_{r-1} \delta_{1} f, \ldots \underbrace{D_{A}^{\Sigma} \cdots D_{B}^{\Sigma}}_{r-r_{N}} \delta_{r_{N}} f) .
$$

Proof. To simplify the notation let us write $\mathcal{U}$ for the bundle

$$
\oplus_{i=0}^{r_{N}} \mathcal{E}_{\underbrace{}_{r-i} \ldots B}\left[\frac{k-n-2 r}{2}\right]
$$

and tf $\mathcal{U}$ for its trace-free subbundle,

$$
\operatorname{tf} \mathcal{U}:=\oplus_{i=0}^{r_{N}} \mathcal{E}_{(\underbrace{A \cdots B}_{r-i})_{0}}\left[\frac{k-n-2 r}{2}\right],
$$

where here and below $(\cdots)_{0}$ indicates the trace-free completely symmetric part over the enclosed indices (and below $(\cdots)$ indicates the completely symmetric part over the enclosed indices). We will write tf $\mathbf{D}$ for the composition of $\mathbf{D}$ with the projection of $\mathcal{U}$ onto tf $\mathcal{U}$.

We will show below that the invariant operator $I$ uniquely determines a homomorphism $H_{0}(I):$ tf $\mathcal{U} \rightarrow \mathcal{V}$ such that $I=H_{0}(I) \circ \mathrm{tfD}$. With this 
established $H(I)$ is given canonically as the composition of $H_{0}(I)$ with the natural projection $\mathcal{U} \rightarrow \mathrm{tf} \mathcal{U}$.

For any $w \in \mathbb{R}$, each bundle $\mathcal{E}_{(A \cdots C)_{0}}^{\Sigma}[w]$ has a natural projection

$$
\mathcal{E}_{(\underbrace{\Sigma}_{v} \underbrace{}_{0})_{0}}^{\Sigma}[w] \rightarrow \mathcal{E}[w+v]
$$

given by

$$
b_{A \cdots C} \mapsto X^{A} \cdots X^{C} b_{A \cdots C} .
$$

It follows from the elementary representation theory of parabolic subgroups that if $\mathcal{S}$ is a subbundle of $\mathcal{E}_{(A \cdots C)_{0}}^{\Sigma}[w]$ that is not in the kernel of this homomorphism then $\mathcal{S}=\mathcal{E}_{(A \cdots C)_{0}}^{\Sigma}[w]$. Now if $f \in \Gamma \mathcal{E}\left[k-\frac{n}{2}\right]$ then

$$
\begin{aligned}
X^{A} \cdots X^{C} \underbrace{D_{(A}^{\Sigma} \cdots D_{C)_{0}}^{\Sigma}}_{r-i} \delta_{i} f & =X^{A} \cdots X^{C} D_{A}^{\Sigma} \cdots D_{C}^{\Sigma} \delta_{i} f \\
& =\prod_{j=0}^{r-i-1}\left(\frac{k-n-2 i-2 j}{2}\right)(2 k-2 i-2 j-1) \delta_{i} f
\end{aligned}
$$

which, for $i \in\left\{0,1, \ldots r_{N}\right\}$, is non-vanishing in general. It follows then that tf $\mathbf{D}$ has vanishing cokernel in tf $\mathcal{U}$.

It follows easily from the definition of the basic (valence 1) tractor bundle and the definition of the tractor metric that, for $i \in\left\{0,1, \ldots, r_{N}\right\}$, each

$$
\mathcal{E}_{(\underbrace{(a \cdots b)}_{j}}^{\Sigma}\left[\frac{k-n-2 i}{2}\right], j=0,1, \ldots, r-i
$$

turns up as a component of a composition factor in the composition series of $\mathcal{E}_{(A \cdots C)_{0}}^{\Sigma}[(k-n-2 r) / 2]$ and that, given a choice of conformal scale, there is a surjection

$$
\mathcal{E}_{(A \cdots C)_{0}}^{\Sigma}\left[\frac{k-n-2 r}{2}\right] \rightarrow \mathcal{E}_{(a \cdots b)}^{\Sigma}\left[\frac{k-n-2 i}{2}\right] .
$$

From classical representation theory and the previous result, the composition of this epimorphism with the operator $D_{(A}^{\Sigma} \cdots D_{C)_{0}}^{\Sigma}$ on $\mathcal{E}[k-i-n / 2]$ gives a differential operator with leading part $\nabla_{(a}^{\Sigma} \cdots \nabla_{c)}^{\Sigma}$. Now in turn using this result, and the normal order of the $\delta_{i}$ operators, it follows that, on $\Sigma$, any linear differential operator on $\mathcal{E}[(k-n) / 2]$, of order $r$ and normal order $r_{N} \leq k-1$, may be written as a composition of a homomorphism $H_{0}(I):$ tf $\mathcal{U} \rightarrow \mathcal{V}$ with tfD. That this homomorphism is unique follows immediately from another use of the result above that the image bundle of $\mathrm{tfD}$ is all of $\mathrm{tf} \mathcal{U}$. (Any non-zero homomorphism $\mathrm{tf} \mathcal{U} \rightarrow \mathcal{V}$ when composed with tfD must yield a nontrivial operator. So if two homomorphisms yield the same composition operator then the homomorphisms agree.) Thus the result is established. 
Remark 7.3. Since a conformally invariant operator $I$ determines the canonical homomorphism $H(I)$ in the lemma it is clear that this homomorphism is conformally invariant. Furthermore, since the operators $D_{A}^{\Sigma} \ldots$ $D_{B}^{\Sigma} \delta_{i}$, for $i=0,1, \ldots, r_{N}$, are linearly independent it is clear that $H(I)$ may be expressed as a direct sum of conformally invariant homomorphisms

$$
H_{i}(I): \underbrace{\mathcal{E}_{A} \cdots C}_{r-i}\left[\frac{k-n-2 r}{2}\right] \rightarrow \mathcal{V},
$$

and $I$ is a sum of conformally invariant operators $I_{i}$, where $I_{i}$ is defined to be the composition of $H_{i}(I)$ with the appropriate component of $\mathbf{D}$. Each $H_{i}(I)$ may be regarded as a conformally invariant section of

$$
\mathcal{E}_{\Sigma}^{A \cdots C}\left[\frac{2 r+n-k}{2}\right] \otimes \mathcal{V}
$$

Given these results the proof of the proposition is now quite straightforward. In the following we will use the terms total order and total normal order as follows. If $\mathrm{Op}_{1} u$ is a linear differential operator of order (resp. normal order) $k_{1}$ and $\mathrm{Op}_{2} u$ is a linear differential operator of order (resp. normal order $k_{2}$ then the bilinear operator $\left(\mathrm{Op}_{1} u\right) \mathrm{Op}_{2} v$ will be said to have total order (resp. total normal order) $k_{1}+k_{2}$. More generally any bilinear operator $\operatorname{Op}(u, v)$ will be said to have total order (resp. total normal order) $\ell$ if there is some expression for it as a sum of products of linear operators such that each product has total order (resp. total normal order) no greater than $\ell$ but no expression as a sum of such products so that each product has total order (resp. total normal order) no greater than $\ell-1$.

Proof of the Proposition. Let $\mathrm{sk}_{\square_{k}}^{\prime}$ be the skew bilinear differential operator on $\mathcal{E}[(k-n) / 2]$ (along $\Sigma$ ) satisfying

$$
\int_{M}\left(u \square_{k} v-v \square_{k} u\right)=-\int_{\Sigma} \operatorname{sk}_{\square_{k}}(u, v)
$$

which is obtained by directly using the formula (20) without any simplification. Some comments on the operator $\mathrm{sk}_{\square_{k}}^{\prime}$ are in order at this point. It is clear that the operator has total order $k-1$. Now note that acting on $u \in \Gamma \mathcal{E}[(k-n) / 2]$ the operator $\square D_{A} \cdots D_{E}$ may be split as follows

$$
\underbrace{D_{A} \cdots D_{E}}_{(k-2) / 2} u=X_{A} \cdots X_{E} K \square_{k} u+W_{A \cdots E} u
$$

where $K \neq 0$ is a constant dependent on the dimension $n$ and $W_{A \cdots E}$ is an invariant order $k-2$ differential operator which vanishes on flat structures, satisfies $D^{E} \cdots D^{A} W_{A \cdots E}=0$ and is such that that the operators

$$
\underbrace{D^{C} \cdots D^{E}}_{j} W_{A \cdots E} \quad j=0,1, \ldots, \frac{k-4}{2}
$$


each have order at most $k-2$. These results follow without difficulty from the definition of the tractor-D given above and the result that on conformally flat structures $\square D_{A} \cdots D_{E} u=X_{A} \cdots X_{E} K \square_{k} u$. Using these observations and (4) it is a straightforward matter to rewrite $\mathrm{sk}_{\square_{k}}^{\prime}$ in the form

$$
\operatorname{sk}_{\square_{k}}^{\prime}(u, v)=\left(E_{(k-2) / 2}^{A \cdots C} u\right) F^{A \cdots C} v-\left(E_{A \cdots C} v\right) F^{A \cdots C} u,
$$

where $E_{A \cdots C}$ and $F^{A \cdots C}$ are conformally invariant differential operators on $\mathcal{E}[(k-n) / 2]$ of orders respectively $k-2$ and $k-1$ and such that the contracted product $\left(E_{A \cdots C} u\right) F^{A \cdots C} v$ has total order $k-1$. Now using the Lemma we can rewrite these in terms of the $D_{A}^{\Sigma}$ operators and the $\delta_{i}$ operators, obtaining

$$
E_{A \cdots C}=\sum_{i=0}^{k-2} E_{(i)_{A \cdots C}}^{L \cdots M} D_{L}^{\Sigma} \cdots D_{M}^{\Sigma} \delta_{i} \text { and } F_{A \cdots C}=\sum_{i=0}^{k-1} F_{(i)}{ }_{A \cdots C}^{L \cdots M} D_{L}^{\Sigma} \cdots D_{M}^{\Sigma} \delta_{i} .
$$

Note that by the Lemma (see also the remark above) the $E_{(i)}{ }_{A \cdots C} \cdots M$, for $i=0, \ldots,(k-2)$, and the $F_{(i)}{ }_{A \cdots C}^{L \cdots M}$, for $i=0, \ldots,(k-1)$, are conformally invariant sections of (boundary manifold) tractor bundles. We now re-express $\operatorname{sk}_{\square_{k}}^{\prime}(u, v)$ using the following observations.

$$
\begin{aligned}
& \left(E_{A \cdots C} u\right) F^{A \cdots C} v \\
& =\left(\sum_{0}^{(k-2) / 2} E_{(i)}{ }_{A \cdots C}^{L \cdots M} D_{L}^{\Sigma} \cdots D_{M}^{\Sigma} \delta_{i} u\right) F^{A \cdots C}+\left(\widetilde{E}_{A \cdots C} u\right) F^{A \cdots C} v,
\end{aligned}
$$

where $\widetilde{E}_{A \cdots C}=\sum_{i=k / 2}^{k-2} E_{(i)}{ }_{A \cdots C}^{L \cdots M} D_{L}^{\Sigma} \cdots D_{M}^{\Sigma} \delta_{i}$. If we similarly split $F_{A \cdots C}$,

$$
\left.F_{A \cdots C}=\sum_{i=0}^{(k-2) / 2} F_{(i)}\right)_{A \cdots C}^{L \cdots M} D_{L}^{\Sigma} \cdots D_{M}^{\Sigma} \delta_{i}+\widetilde{F}_{A \cdots C}
$$

then we obtain

$$
\begin{aligned}
\left(E_{A \cdots C} u\right) F^{A \cdots C} v= & \left(\sum_{i=0}^{(k-2) / 2} E_{(i)}{ }_{A \cdots C}^{L \cdots M} D_{L}^{\Sigma} \cdots D_{M}^{\Sigma} \delta_{i} u\right) F^{A \cdots C} v \\
& +\left(\widetilde{E}^{A \cdots C} u\right) \sum_{i=0}^{(k-2) / 2} F_{(i)}{ }_{A \cdots C}^{L \cdots M} D_{L}^{\Sigma} \cdots D_{M}^{\Sigma} \delta_{i} v \\
& +\left(\widetilde{E}_{A \cdots C} u\right) \widetilde{F}^{A \cdots C} v .
\end{aligned}
$$

Now the operator $\left(\widetilde{E}_{A \cdots C} u\right) \widetilde{F}_{A \cdots C} v$ must vanish since otherwise it is of total order strictly greater than $k-1$; this is not possible since it is clearly linearly independent of the other operators on the right hand side of the above 
display. Thus by repeated use of (19) (applied to the boundary manifold operator $\left.D_{A}^{\Sigma}\right)$ it is clear that we have

$$
\left(E_{A \cdots C} u\right) F^{A \cdots C} v=\sum_{0}^{(k-2) / 2}\left[\left(\delta_{i} u\right) F_{i} v+\left(E_{i} u\right) \delta_{i} v\right]+\text { a } \nabla^{\Sigma} \text { divergence }
$$

where

$$
F_{i}=D_{M}^{\Sigma} \cdots D_{L}^{\Sigma} E_{(i)}{ }_{A \cdots C}^{L \cdots M} F^{A \cdots C}
$$

and

$$
E_{i}=D_{M}^{\Sigma} \cdots D_{L}^{\Sigma} F_{(i)}{ }_{A \cdots C}^{L \cdots M} \widetilde{E}^{A \cdots C} .
$$

Thus defining the operators $\delta_{i}^{\prime}$ and $\delta_{k-1-i}^{\prime}$, for $i=0,1, \ldots,(k-2) / 2$, by

$$
\delta_{i}^{\prime}:=\delta_{i} \text { and } \delta_{k-1-i}^{\prime}:=\left(F_{i}-E_{i}\right)
$$

we have

$$
\operatorname{sk}_{\square_{k}}^{\prime}(u, v)=\operatorname{sk}_{\square_{k}}(u, v)+\text { a } \nabla^{\Sigma} \text { divergence, }
$$

where

$$
\operatorname{sk}_{\square_{k}}(u, v)=\sum_{i=0}^{(k-2) / 2}\left[\left(\delta_{i}^{\prime} u\right) \delta_{k-1-i}^{\prime} v-\left(\delta_{i}^{\prime} v\right) \delta_{k-1-i}^{\prime} u\right] .
$$

Now the proposition follows from the following points:

- Each $\delta_{i}^{\prime}, i=0, \ldots,(k-1)$ is conformally invariant and canonical. (In particular they are independent of any choice of extension of $N^{a}$ off $\Sigma$.) This is immediately clear from their construction via natural conformally invariant operators which in turn are defined without a choice of extension of $N^{a}$ off $\Sigma$. (Note also the $E_{i}$ and $F_{i}$ above are in fact separately conformally invariant.)

- Up to a non-zero scalar factor, each operator $\delta_{i}^{\prime}, i=0, \ldots, k-1$ is of the form

$$
\delta_{i}^{\prime}=\left(\nabla_{N}\right)^{i}+\text { lower normal order terms. }
$$

Here and below we have assumed some arbitrary extension of $N^{a}$ off $\Sigma$. This result can be seen as follows. For each $k$ the operator $\square_{k}$ has the form $\Delta^{k / 2}+$ lower order terms, up to a non-zero scale factor. Therefore it is clear that up to $\nabla^{\Sigma}$-divergences and terms of total order $\leq k-1, \mathrm{sk}_{\square_{k}}$ must agree with $\mathrm{sk}_{\Delta^{k / 2}}$. Thus the leadingnormal-order part of $\mathrm{sk}_{\square_{k}}$ is (again, up to a non-zero-scale factor) $\sum_{i=0}^{(k-2) / 2}\left[\left(\left(\nabla_{N}\right)^{i} u\right)\left(\nabla_{N}\right)^{k-1-i} v-\left(\left(\nabla_{N}\right)^{i} v\right)\left(\nabla_{N}\right)^{k-1-i} u\right]$. (This is clear since this term cannot be changed by the adding of $\nabla^{\Sigma}$-divergences or lower total order terms to the operator $\mathrm{sk}_{\Delta^{k / 2}}$.) Now using order and weight considerations, the claim here follows. 
- If we take $\delta_{\mathbf{m}}^{\prime}=\left(\delta_{m_{1}}^{\prime}, \ldots, \delta_{m_{k / 2}}^{\prime}\right)$ with $\mathbf{m}=\left(m_{1}, \ldots, m_{k / 2}\right)$ as in (17), it is immediate from the form of $\mathrm{sk}_{\square_{k}}$, as displayed in (25), that $\left(\square_{k}, \delta_{\mathbf{m}}^{\prime}\right)$ is formally self-adjoint.

Let $\delta_{\mathbf{m}}=\left(\delta_{m_{1}}, \ldots, \delta_{m_{k / 2}}\right)$, and let $\delta_{\mathbf{m}}^{\prime}=\left(\delta_{m_{1}}^{\prime}, \ldots, \delta_{m_{k / 2}}^{\prime}\right)$.

Theorem 7.4. Let $k$ be a positive even integer, and if $n$ is even, suppose that $k<n$. Then the boundary problems $\left((-1)^{k / 2} \square_{k}, \delta_{\mathbf{m}}\right)$ and $\left((-1)^{k / 2} \square_{k}\right.$, $\left.\delta_{\mathbf{m}}^{\prime}\right)$ satisfy the LS condition for the choices $\mathbf{m}=\mathbf{m}_{\mathrm{D}}=(0,2, \ldots, k-2), \mathbf{m}=$ $\mathbf{m}_{\mathrm{N}}=(1,3, \ldots, k-1)$, and $\mathbf{m}=\mathbf{m}_{0}=(0,1, \ldots, k / 2-1)$. In addition, when $n$ is even, the LS condition holds for the boundary problems $\left((-1)^{n / 2} \square_{n}, \delta_{\mathbf{m}}\right)$ with $\mathbf{m}=\mathbf{m}_{\mathrm{D}}$ and with $\mathbf{m}=\mathbf{m}_{0}$.

Proof. The statements about $\mathbf{m}_{0}$ are clear from (26), together with the fact that (up to non-vanishing scale) $\delta_{i}$ and $\delta_{i}^{\prime}$ differ only at lower normal order. The polynomials in the LS condition have degrees $0,1, \ldots, k / 2-1$ in $\tau$ respectively, and thus are linearly independent in $\mathbb{C}[\tau] / \mathbb{C}[\tau] M^{+}(\tau)$, since $\operatorname{deg} M^{+}=k / 2$. For $\mathbf{m}_{\mathrm{D}}$ and $\mathbf{m}_{\mathrm{N}}$, we need to use Lemma 6.3. First notice that by Remark 6.2 , the principal parts of the boundary operators in the ordinary Riemannian iterated Dirichlet and Neumann problems, $\left((-\Delta)^{k / 2}, S_{\mathbf{m}_{\mathrm{D}}}\right)$ and $\left((-\Delta)^{k / 2}, S_{\mathbf{m}_{\mathrm{N}}}\right)$, must take the form (14) with $c_{q}=1$. After normalization, (26) shows that a similar statement holds for the problems $\left((-1)^{k / 2} \square_{k}, \delta_{\mathbf{m}_{\mathrm{D} \text { or N }}}\right)$ and $\left((-1)^{k / 2} \square_{k}, \delta_{\mathbf{m}_{\mathrm{D} \text { or N }}}^{\prime}\right)$, when these problems are defined. Thus $\delta_{\mathbf{m}_{\text {Dor N }}}^{\prime}\left(\right.$ or $\delta_{\mathbf{m}_{\text {D or N }}}$ ) is related to $S_{\mathbf{m}_{\text {D or N }}}$ as $B_{\mathbf{m}}^{\prime}$ is to $B_{\mathbf{m}}$ in Lemma 6.3. (An important point is that the parities of the $m_{j}$ agree, so we can adjust at lower order using linear combinations with tangential differential operator coefficients.) The problems $\left((-\Delta)^{k / 2}, S_{\mathbf{m}_{\mathrm{D} \text { or N }}}\right)$ satisfy the LS condition by Remark 6.1. Thus Lemma 6.3 gives the conclusion.

\section{Construction of the non-local invariant operators.}

So far, we have constructed conformally invariant boundary problems $\left((-1)^{k / 2} \square_{k}, \delta_{\mathbf{m}}\right)$ for all even positive integers $k$, except when $n$ is even and $k>n$ (Theorem 5.1). We have also constructed conformally invariant, formally self-adjoint modifications of these problems, the $\left((-1)^{k / 2} \square_{k}, \delta_{\mathbf{m}}^{\prime}\right)$, as long as $\mathbf{m}$ satisfies (17), and $k$ is further restricted by excluding the case in which $n$ is even and $k=n$ (Proposition 7.1). Furthermore, we have shown that these problems, in the cases $\mathbf{m}=\mathbf{m}_{\mathrm{D}}, \mathbf{m}_{\mathrm{N}}$, or $\mathbf{m}_{0}$, are elliptic in the sense of the Lopatinski-Shapiro condition based on a properly elliptic interior differential operator (Theorem 7.4). The fact that the LS condition is satisfied allows application of the general results of Proposition 6.4 on existence and uniqueness of solutions (given vanishing deficiency indices), and on elliptic regularity. These conformally invariant problems may be called 
the conformal iterated Dirichlet problem (in the case $\mathbf{m}=\mathbf{m}_{\mathrm{D}}$ ), the conformal iterated Neumann problem (in the case $\mathbf{m}=\mathbf{m}_{\mathrm{N}}$ ), and the conformal generalized Dirichlet problem (in the case $\mathbf{m}=\mathbf{m}_{0}$ ).

By Proposition 6.4, the problems

$$
\left((-1)^{k / 2} \square_{k}, \delta_{\mathbf{m}_{\text {D or N or } 0}^{\prime}}^{\prime}\right),
$$

when defined, have unique smooth solutions for all choices of smooth data, provided they have vanishing null space. "Vanishing null space" for the even (resp. odd) problem of this type is the condition that there is no non-trivial smooth density $\psi$ for which

$$
\square_{k} \psi=0 \text { and } \delta_{m_{j}}^{\prime} \psi=0 \text {, all } m_{j} .
$$

For the problems which are not known to be formally self-adjoint (those involving the $\delta_{\mathbf{m}}$ as opposed to the $\delta_{\mathbf{m}}^{\prime}$ ) we must assume that a realization of the boundary value problem as an operator is bijective.

Note that for the construction of the classical Dirichlet-to-Neumann operator, only the Dirichlet problem needs to be uniquely solvable - one solves the Dirichlet problem, and then computes Neumann data of the solution. The same principle applies, of course, to the problems we study - a "source problem" needs to be uniquely solvable; a "target problem" is also involved, but its solvability properties are immaterial. (We will, however, make use of the ellipticity of the target problem.) We shall now construct our conformal, higher-order generalizations of the Dirichlet-to-Neumann operator.

Let $M$ be an $n$-dimensional conformal manifold of positive definite metric signature, with smooth boundary $\Sigma$. Suppose that $k$ is even and, if $n$ is even, suppose that $k<n$. Let $\mathbf{m}=\mathbf{m}_{\mathrm{D} \text { or N or } 0}$, and suppose that the problem $\left(\square_{k}, \delta_{\mathbf{m}}^{\prime}\right)$ has vanishing null space. Take a density $u$ on $\Sigma$, and boundary data

$$
\delta_{m_{j}}^{\prime} \psi=u, \quad \delta_{m_{i}}^{\prime} \psi=0 \text { for all } i \neq j,
$$

where $j$ is a chosen element of $\{1, \ldots, k / 2\}$. Let $E_{k, m_{j}}^{\prime}$ be the solution operator for the system $\square_{k} \psi=0$ with (28); by construction, $E_{k, m_{j}}^{\prime}$ is an invariant operator carrying $\mathcal{E}_{\Sigma}\left[\frac{k-n-2 m_{j}}{2}\right]$ to $\mathcal{E}\left[\frac{k-n}{2}\right]$. We can now take $E_{k, m_{j}}^{\prime} u$ and apply $\delta_{\ell}^{\prime}$ (or $\delta_{\ell}$ ). ( $\ell$ need not be one of the normal orders in $\mathbf{m}$.) Composing,

$$
\mathcal{E}_{\Sigma}\left[\frac{k-n-2 m_{j}}{2}\right] \stackrel{E_{k, m_{j}}^{\prime}}{\longrightarrow} \mathcal{E}\left[\frac{k-n}{2}\right] \stackrel{\delta_{\ell}^{\prime}}{\longrightarrow} \mathcal{E}_{\Sigma}\left[\frac{k-n-2 \ell}{2}\right],
$$

we obtain invariant operators

$$
P_{k, \mathbf{m}, m_{j}, \ell}^{\prime}: \mathcal{E}_{\Sigma}\left[\frac{k-n-2 m_{j}}{2}\right] \rightarrow \mathcal{E}_{\Sigma}\left[\frac{k-n-2 \ell}{2}\right] .
$$

We have established the following theorem: 
Theorem 8.1. Let $M$ be an $n$-dimensional conformal manifold of positive definite metric signature, with smooth boundary $\Sigma$. Suppose that $k$ is even

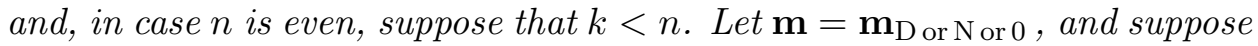
that the problem $\left(\square_{k}, \delta_{\mathbf{m}}^{\prime}\right)$ has vanishing null space. Suppose that $\ell<k$. Then there exist canonical conformally invariant operators

$$
P_{k, \mathbf{m}, m_{j}, \ell}^{\prime}: \mathcal{E}_{\Sigma}\left[\frac{k-n-2 m_{j}}{2}\right] \rightarrow \mathcal{E}_{\Sigma}\left[\frac{k-n-2 \ell}{2}\right] .
$$

We caution that many of these operators (those for which $m_{j}+\ell \neq k-1$ ) are "morally zero", in the sense that they vanish for the standard conformal class on the unit ball. (See Theorem 8.4 and its proof below. There we show vanishing of these operators on the unit hemisphere, which is in the conformal class of the unit ball.) As a consequence, in the case of general $M$, though the construction of $P_{k, \mathbf{m}, m_{j}, \ell}^{\prime}$ appears to produce a pseudo-differential operator of order $\ell-m_{j}$, Theorem 8.4 will show that for $m_{j}+\ell \neq k-1$, the order of this operator is actually at most $\ell-m_{j}-1$.

We shall show below that the operator $P_{k, \mathbf{m}, m_{j}, k-1-m_{j}}^{\prime}$ has principal part $\left(-\Delta_{\Sigma}\right)^{\left(k-1-2 m_{j}\right) / 2}$, up to multiplication by a non-zero constant (Theorem 8.4), and is formally self-adjoint (Theorem 8.5).

Theorem 8.1 did not provide a conclusion for $n$ even and $k=n$, owing to the fact that we have not constructed formally self-adjoint boundary problems in this case. Making a stronger assumption, however, we can cover this case.

Theorem 8.2. Let $(M, \Sigma)$ be as in Theorem 8.1, and suppose that $k$ is even and

$$
\begin{aligned}
& n \text { is odd and } \mathbf{m}=\mathbf{m}_{\mathrm{D} \text { or } \mathrm{N} \text { or } 0}, \text { or } \\
& n \text { is even, } k<n, \text { and } \mathbf{m}=\mathbf{m}_{\mathrm{D} \text { or } \mathrm{N} \text { or } 0}, \text { or } \\
& n \text { is even, } k=n, \text { and } \mathbf{m}=\mathbf{m}_{\mathrm{D} \text { or } 0} .
\end{aligned}
$$

Suppose further that the problem

$$
\square_{k} \psi=0, \quad \delta_{\mathbf{m}} \psi \text { prescribed }
$$

is uniquely solvable. In case $n$ is even, suppose that $\ell \leq n-2$. Then there exist canonical, conformally invariant operators

$$
P_{k, \mathbf{m}, m_{j}, \ell}: \mathcal{E}_{\Sigma}\left[\frac{k-n-2 m_{j}}{2}\right] \rightarrow \mathcal{E}_{\Sigma}\left[\frac{k-n-2 \ell}{2}\right] .
$$

The proof of Theorem 8.1 above makes it clear how Theorem 8.2 follows from the stronger assumption; here we use the operators $\delta_{\ell}$, as opposed to the $\delta_{\ell}^{\prime}$, together with solution operators $E_{k, m_{j}}$ based on the (unprimed) $\delta_{\ell}$. Again, one cannot expect the $P_{k, \mathbf{m}, m_{j}, \ell}$ to be interesting except in the case $m_{j}+\ell=k-1$.

If we treat $\Sigma$ as the fundamental object, we may improve Theorem 8.1 by weakening our assumption involving (27) somewhat. Fixing a conformal structure on $\Sigma$, there are many choices for an extending conformal structure 
on $M$, and in fact many topological types of $M$ admitting $\Sigma$ as boundary. Even if our boundary problem has a kernel on $M$, it need not have one for a different choice $M^{\prime}$ :

Corollary 8.3. If $\Sigma$ is a compact conformal manifold of dimension $n^{\Sigma} \geq$ 2 , and $M$ is a compact conformal manifold with boundary $\Sigma^{\prime}$ conformally diffeomorphic to $\Sigma$, and (27) has no non-trivial solutions on $\left(M, \Sigma^{\prime}\right)$, then conformally invariant operators with the properties asserted in Theorem 8.1 exist on $\Sigma$, subject to that theorem's constraints on $(n, k, \mathbf{m}, \ell)$.

There is, of course, an analogous corollary to Theorem 8.2.

The Dirichlet-to-Neumann operator is known to be a pseudo-differential operator with leading symbol $|\eta|=\left(g_{\Sigma}^{a b} \eta_{a} \eta_{b}\right)^{1 / 2}[46]$. (Here we use $\eta$ to denote an indeterminate vector from $\mathcal{E}_{a}^{\Sigma}$, to distinguish it from an indeterminate vector $\xi$ from $\mathcal{E}_{a}$, and in harmony with the notation of Section 6.) In fact, its coordinate-dependent total symbol has a polyhomogeneous expansion

$$
\sigma(\mathcal{B})(x, \eta) \sim|\eta|+\sum_{k=0}^{\infty} p_{-k}(x, \eta),
$$

where $p_{-k}$ is homogeneous in $\eta$ of order $-k$; i.e., for $\lambda>0$,

$$
p_{-k}(x, \lambda \eta)=\lambda^{-k} p_{-k}(x, \eta) .
$$

One might imagine that each operator $P_{k, \mathbf{m}, m_{j}, \ell}$ has similar properties, and in particular, has leading symbol a non-zero constant times $|\eta|^{\ell-m_{j}}$. This, however, is true if and only if the parameters satisfy the condition

$$
m_{j}+\ell=k-1,
$$

as we shall now show. Note that for the previously known operators, namely the conformal Dirichlet-to-Robin and Robin-to-Dirichlet operators when they exist, (29) is satisfied: $k-1=1$, and $\left\{m_{j}, \ell\right\}=\{0,1\}$.

Theorem 8.4. Each operator $P_{k, \mathbf{m}, m_{j}, \ell}^{\prime}$ constructed in Theorem 8.1 is pseudodifferential. The coordinate-dependent total symbol of $P_{k, \mathbf{m}, m_{j}, \ell}^{\prime}$ has a polyhomogeneous expansion

$$
\sigma\left(P_{k, \mathbf{m}, m_{j}, \ell}^{\prime}\right)(x, \eta) \sim c_{k, \mathbf{m}, m_{j}, \ell}|\eta|^{\ell-m_{j}}+\sum_{k=0}^{\infty} p_{\ell-m_{j}-k}(x, \eta),
$$

where $|\eta|^{2}=g^{a b} \eta_{a} \eta_{b}=g_{\Sigma}^{a b} \eta_{a} \eta_{b}$, each $p_{d}$ is homogeneous in $\eta$ of order $d$, and $c_{k, \mathbf{m}, m_{j}, \ell}$ is a universal (independent of $(M, \Sigma)$ ) constant. Let $\left(\mathbf{m}, \mathbf{m}^{\prime}\right)=$ $\left(\mathbf{m}_{\mathrm{D}}, \mathbf{m}_{\mathrm{N}}\right)$ or $\left(\mathbf{m}_{\mathrm{N}}, \mathbf{m}_{\mathrm{D}}\right)$, and suppose

$$
m_{j} \in \mathbf{m}, \quad \ell \in \mathbf{m}^{\prime} .
$$

Then $c_{k, \mathbf{m}, m_{j}, \ell}$ is non-zero if and only if $m_{j}+\ell=k-1$. In particular, $P_{k, \mathbf{m}, m_{j}, \ell}^{\prime}$ has order $\ell-m_{j}$ if and only if $m_{j}+\ell=k-1$. 
Proof. The pseudo-differential character of $P_{k, \mathbf{m}, m_{j}, \ell}^{\prime}$ and the existence of a polyhomogeneous expansion with the orders stated follows just as for the classical Dirichlet-to-Neumann operator.

Let $x$ be a fixed but arbitrary point of $M$. Because the construction of the operators is equivariant with respect to the structure group $\mathrm{O}\left(n^{\Sigma}\right)$, the leading symbol is $\mathrm{O}\left(n^{\Sigma}\right)$-invariant. Thus, when restricted to the unit sphere in $\mathcal{E}_{a}^{\Sigma}$, it is constant. Because of its homogeneity, it thus has the form $f(x)|\eta|^{\ell-m_{j}}$. On the other hand, the formula for the leading symbol of the solution operator (our $E_{k, m_{j}}$ ) given in [36], (6.4.19) shows that, for tangential $\eta_{a}$, this leading symbol depends only on the roots $\tau_{j}^{+}$(as in Section 6, with $A=(-1)^{k / 2} \square_{k}$ ) and on $|\eta|$. Since $(-1)^{k / 2} \square_{k}$ has leading symbol $|\xi|^{k}$, these $\tau_{j}^{+}$are all $\sqrt{-1}|\eta|$, and thus depend only on $|\eta|$. The formula as given in [36] is good for problems of type $\mathbf{m}_{0}$, but [36], Section 6.5 indicates the extension of the formula to the case of general $\mathbf{m}$. This shows that $f(x)$ is a constant function, and in fact a universal constant $c_{k, \mathbf{m}, m_{j}, \ell}$.

Now suppose that $\left(\mathbf{m}, \mathbf{m}^{\prime}\right)=\left(\mathbf{m}_{\mathrm{D}}, \mathbf{m}_{\mathrm{N}}\right)$ or $\left(\mathbf{m}_{\mathrm{N}}, \mathbf{m}_{\mathrm{D}}\right)$, and that we are in the situation of (30). Consider the example in which $M$ is the $n$-dimensional unit hemisphere $H^{n}$ with its standard conformal class (containing the round $n$-sphere metric), and the boundary $\Sigma$ is the equator $S^{n-1}$, inheriting its standard conformal class under pullback by the inclusion. This conformal class contains the standard round $(n-1)$-sphere metric. We first need to know that the construction of the operators $P_{k, \mathbf{m}, m_{j}, \ell}^{\prime}$ applies to this case; i.e., that the kernel of $\left(\square_{k}, \delta_{\mathbf{m}}^{\prime}\right)$ vanishes on the hemisphere. First note that

$$
\begin{aligned}
(-1)^{k / 2}\left(\square_{k}\right)_{S^{n}} & =\frac{\Gamma\left(A+\frac{k}{2}+\frac{1}{2}\right)}{\Gamma\left(A-\frac{k}{2}+\frac{1}{2}\right)} \\
& =\left(A-\frac{k-1}{2}\right) \ldots\left(A-\frac{1}{2}\right)\left(A+\frac{1}{2}\right) \ldots\left(A+\frac{k-1}{2}\right),
\end{aligned}
$$

where

$$
A=\sqrt{-\Delta_{S^{n}}+\left(\frac{n-1}{2}\right)^{2}} .
$$

(See [8], Remark 2.23.) In particular, the spherical harmonic decomposition of functions on $S^{n}$ gives a spectral resolution of $(-1)^{k / 2} \square_{k}$ there; the eigenvalues are

$$
\lambda_{j}=\frac{2 j+n-k}{2}\left(\frac{2 j+n-k}{2}+1\right) \ldots\left(\frac{2 j+n+k}{2}-1\right) .
$$

If $n$ is odd, these are products of proper half-integers; if $n$ is even, the bottom eigenvalue is

$$
\frac{n-k}{2}\left(\frac{n-k}{2}+1\right) \ldots\left(\frac{n+k}{2}-1\right)>0 .
$$

In particular, all eigenvalues are non-zero. 
We now claim that on $H^{n}$, the operator $\delta_{\ell}^{\prime}$ has the form

$$
\begin{cases}c_{\ell}\left(\nabla_{N}\right)^{\ell}+D_{\ell-2}\left(\nabla_{N}\right)^{\ell-2}+\cdots+D_{0} & (\ell \text { even }), \\ c_{\ell}\left(\nabla_{N}\right)^{\ell}+D_{\ell-2}\left(\nabla_{N}\right)^{\ell-2}+\cdots+D_{1} \nabla_{N} & (\ell \text { odd }),\end{cases}
$$

where $c_{\ell}$ is a non-zero constant, and the $D_{i}$ are tangential differential operators. The leading normal order term has already been established for general $M$. The hemisphere is locally symmetric (i.e., has $\nabla R=0$ ) with totally geodesic boundary (vanishing second fundamental form $L$ ). A check of parity considerations in the local invariant theory shows that under these circumstances, there can be no terms in $\delta_{\ell}^{\prime}$ with a normal derivative count of the "wrong" (opposite to $\ell$ ) parity. (Coefficients of terms with the wrong parity must involve the second fundamental form and/or its derivatives, odd derivatives of the Riemann tensor, or the quantity $R_{a b c N}$, where $a, b, c$ are concrete tangential indices and $N$ the unit normal index. However, in general, $R_{a b c N}=\nabla_{b}^{\Sigma} L_{a c}-\nabla_{a}^{\Sigma} L_{b c}$ on $\Sigma$. Thus $R_{a b c N}$ vanishes in the present setting.)

As a consequence, the system

$$
\left(\delta_{m}^{\prime} u=0\right)_{m \in \mathbf{m}}
$$

is equivalent to the system

$$
\left(\left(\nabla_{N}\right)^{m} u=0\right)_{m \in \mathbf{m}} .
$$

Indeed, using (32), we get (33) by induction on $m$ using (31). Given (33), (32) is immediate by (31). Thus we may replace the system $\square_{k} u=0$ with (32) with the system $\square_{k} u=0$ with (33). By the $\mathrm{O}(n+1)$ invariance of $\square_{k}$ on $S^{n}$, we can write explicit spectral resolutions of the problems $\square_{k}$ with (33): The eigensections are the $\left.f\right|_{H^{n}}$ for spherical harmonics $f$ which are odd (resp. even) under reflection across the equator when $\mathbf{m}=\mathbf{m}_{\mathrm{D}}$ (resp. $\mathbf{m}=\mathbf{m}_{\mathrm{N}}$ ). (The spherical harmonics give a complete orthonormal set in $L^{2}\left(S^{n}\right)$, and thus in $L^{2}\left(H^{n}\right)$.) Equivalently, these are spherical harmonics of odd (resp. even) degree; i.e., degree of parity opposite to that of the elements of $\mathbf{m}$ ). We computed above, however, that the eigenvalue of $\square_{k}>0$ on each spherical harmonic degree is non-zero. This shows that the systems in question have no kernel, and thus the choice $(M, \Sigma)=\left(H^{n}, S^{n-1}\right)$ provides us with conformally invariant operators $P_{k, \mathbf{m}, m_{j}, \ell}^{\prime}$ on densities over $S^{n-1}$.

Conformally invariant operators are in particular invariant under the identity component of the conformal diffeomorphism group of $S^{n-1}$, viz. $\mathrm{SO}_{0}(n, 1)$. Thus the realization of $P_{k, \mathbf{m}, m_{j}, \ell}^{\prime}$ on the sphere is intertwining between the spherical principal series representations carried by the source and target spaces,

$$
\mathcal{E}_{S^{n-1}}\left[\frac{k-n-2 m_{j}}{2}\right] \quad \text { and } \quad \mathcal{E}_{S^{n-1}}\left[\frac{k-n-2 \ell}{2}\right] .
$$


For this intertwinor to be non-zero, the values of the Casimir operator of $\mathfrak{s o}(n, 1)$ in these two representations,

$$
\left(\frac{k-n-2 m_{j}}{2}+\frac{n-1}{2}\right)^{2}-\left(\frac{n-1}{2}\right)^{2} \quad \text { and } \quad\left(\frac{k-n-2 \ell}{2}+\frac{n-1}{2}\right)^{2}-\left(\frac{n-1}{2}\right)^{2},
$$

must agree. (See, e.g., [14], Lemma 4.1 and Example 3.a.) This condition is equivalent to

$$
k-1-2 \ell= \pm\left(k-1-2 m_{j}\right) .
$$

The two solutions are $\ell=m_{j}$, and (29). The first of these is not available to us, since $\mathbf{m}_{\mathrm{D}} \cap \mathbf{m}_{\mathrm{N}}=\emptyset$, and thus on $\left(H^{n}, S^{n-1}\right)$, we have $P_{k, \mathbf{m}, m_{j}, \ell}^{\prime} \neq$ $0 \Rightarrow(29)$. In particular, the universal constants $c_{k, \mathbf{m}, m_{j}, \ell}$ vanish when (29) is not satisfied.

We now claim that $P_{k, \mathbf{m}, m_{j}, k-1-m_{j}}^{\prime} \neq 0$ on $\left(H^{n}, S^{n-1}\right)$. For if $P_{k, \mathbf{m}, m_{j}, k-1-m_{j}}^{\prime}=0$, the vanishing of the $P_{k, \mathbf{m}, m_{j}, \ell}^{\prime}$ for $\ell \neq k-1-m_{j}$ established above shows that for all $u \in \Gamma \mathcal{E}\left[\frac{k-n-2 m_{j}}{2}\right]$, all the $\mathbf{m}^{\prime}$ data of $E_{k, m_{j}}^{\prime} u$ vanish. This is a contradiction, as it puts the infinite-dimensional space $E_{k, m_{j}}^{\prime} \Gamma \mathcal{E}\left[\frac{k-n-2 m_{j}}{2}\right]$ inside the space $\operatorname{ker}\left(\square_{k}, \delta_{\mathbf{m}^{\prime}}^{\prime}\right)$ which, as the kernel of an elliptic boundary problem, is finite dimensional. (In fact, this last space is 0 , as shown above. Since the boundary data of a section may be recovered from the section itself, $E_{k, m_{j}}^{\prime}$ is injective. $E_{k, m_{j}}^{\prime} \Gamma \mathcal{E}\left[\frac{k-n-2 m_{j}}{2}\right]$ is infinite dimensional because it is the injective image of $\Gamma \mathcal{E}\left[\frac{k-n-2 m_{j}}{2}\right]$.) However, there is a unique (up to a constant factor) principal series intertwinor between the line bundles in question when (29) holds, viz. (1) for $2 r=\ell-m_{j}$. Since $\ell-m_{j}=k-1-2 m_{j}$ is odd, this takes the form

$$
A_{k-1-2 m_{j}}=\left\{\begin{array}{l}
\left(B-\frac{k-2-2 m_{j}}{2}\right) \ldots(B-1) B(B+1) \ldots\left(B+\frac{k-2-2 m_{j}}{2}\right), \\
2 m_{j}<k-1, \\
{\left[\left(B+\frac{k-2-2 m_{j}}{2}\right) \ldots(B-1) B(B+1) \ldots\left(B-\frac{k-2-2 m_{j}}{2}\right)\right]^{-1},} \\
2 m_{j}>k-1 .
\end{array}\right.
$$

(See [14], Example 3.a for the derivation of (1). Uniqueness is a special case of [9], Lemma 3.5. The formula (37) first appeared in [8], Remark 2.23.) Thus $P_{k, \mathbf{m}, m_{j}, k-1-m_{j}}^{\prime}=a_{k, \mathbf{m}, m_{j}, k-1-m_{j}} A_{\ell-m_{j}}$, with $a_{k, \mathbf{m}, m_{j}, k-1-m_{j}} \neq 0$. The leading symbol of $A_{\ell-m_{j}}$ is $|\eta|^{\ell-m_{j}}$, and thus the universal constant $c_{k, \mathbf{m}, m_{j}, k-1-m_{j}}$ is identical with $a_{k, \mathbf{m}, m_{j}, k-1-m_{j}}$.

Theorem 8.5. The operators $P_{k, \mathbf{m}, m_{j}, k-1-m_{j}}^{\prime}$ of Theorem 8.1 are formally self-adjoint. 
Proof. Proof If $u, v \in \Gamma \mathcal{E}_{\Sigma}\left[\frac{k-n-2 m_{j}}{2}\right]$, then by (23), (24), and (25),

$$
\begin{aligned}
& \int_{\Sigma}\left\{u P_{k, \mathbf{m}, m_{j}, k-1-m_{j}}^{\prime} v-\left(P_{k, \mathbf{m}, m_{j}, k-1-m_{j}}^{\prime} u\right) v\right\} \\
& =\int_{\Sigma}\left\{\left(\delta_{m_{j}}^{\prime} E_{k, m_{j}}^{\prime} u\right) \delta_{k-1-m_{j}}^{\prime} E_{k, m_{j}}^{\prime} v-\left(\delta_{k-1-m_{j}}^{\prime} E_{k, m_{j}}^{\prime} u\right) \delta_{m_{j}}^{\prime} E_{k, m_{j}}^{\prime} v\right\} \\
& =\int_{\Sigma} \operatorname{sk}_{\square_{k}}^{\prime}\left(E_{k, m_{j}}^{\prime} u, E_{k, m_{j}}^{\prime} v\right) \\
& =-\int_{M}\left\{\left(E_{k, m_{j}}^{\prime} u\right) \square_{k} E_{k, m_{j}}^{\prime} v-\left(\square_{k} E_{k, m_{j}}^{\prime} u\right) E_{k, m_{j}}^{\prime} v\right\} \\
& =0
\end{aligned}
$$

since $\square_{k} E_{k, m_{j}}^{\prime} u=\square_{k} E_{k, m_{j}}^{\prime} v=0$.

\section{Final remarks.}

The operators we construct have an interesting relation to the Poisson transform on symmetric spaces [33]. (We thank Gestur Ólafsson and Bent Ørsted for enlightening discussions on this point.) Given a semisimple Lie group $G$, the Poisson transform $\mathcal{P}_{\lambda}$ carries functions on the generalized boundary $G / P$ of the symmetric space $G / K$ ( $K$ a maximal compact subgroup, $P$ a minimal parabolic subgroup). More precisely, for $\lambda \in \mathfrak{a}^{*}$ (where $\mathfrak{k}+\mathfrak{a}+\mathfrak{n}$ is the Iwasawa decomposition), we get a transform $\mathcal{P}_{\lambda}$ carrying the spherical principal series representation space with $\mathfrak{a}^{*}$-parameter $\lambda$ to a joint eigenspace of the invariant differential operators on $G / K$; the eigenvalues involved being determined by $\lambda$.

In the relevant special case of this situation, $G=\mathrm{SO}_{0}(n, 1)$, the symmetric space $G / K$ is the $n$-dimensional hyperboloid $\mathbb{H}^{n}$, and $G / P$ is the $(n-1)$ dimensional sphere $S^{n-1}$. Assigning the standard metrics (of | sectional curvature| one) to both spaces, the pair $\left(\mathbb{H}^{n}, S^{n-1}\right)$, as a manifold with boundary, now has the same conformal structure as our previous examples $\left(B^{n}, S^{n-1}\right)$ and $\left(H^{n}, S^{n-1}\right)$. The metric pair $\left(g_{\mathbb{H}^{n}}, g_{S^{n-1}}\right)$ cannot be related to the pair $\left(g_{B^{n}}, g_{S^{n-1}}\right)$ by a smooth conformal factor, however, as $g_{B^{n}}=\Omega^{2} g_{\mathbb{H}^{n}}$ with $\Omega \rightarrow \infty$ as we approach the boundary; this, in fact, is a commonplace occurrence in the setting of conformal structure on manifolds with boundary. One way to handle this and still describe things in terms of a scale (choice of Riemannian metric) is to think of the Poisson transform as a composition

boundary data on $S^{n-1} \stackrel{\text { extension op. }}{\longrightarrow}$ densities on $B^{n}$ $\stackrel{\text { rescaling }}{\longrightarrow}$ densities on $\mathbb{H}^{n}$,

where in the last step we have exploited the conformal invariance of the interior operators. 
Consider the interior operator $\square_{k}$, where, in case $n$ is even, we assume $k<n$. From [8], Remark 2.23, we know this operator on $S^{n}$ to be

$$
\prod_{a=1}^{k / 2}\left(-\Delta-\left(a-\frac{1}{2}\right)^{2}+\left(\frac{n-1}{2}\right)^{2}\right) \text {. }
$$

The constants involved come from curvatures in the arbitrarily curved expression for $\square_{k}$, so they are sign-reversed upon passage from $S^{n}$ to $\mathbb{H}^{n}$. Thus

$$
\square_{k}=\prod_{a=1}^{k / 2}\left(-\Delta+\left(a-\frac{1}{2}\right)^{2}-\left(\frac{n-1}{2}\right)^{2}\right) \quad \text { on } \mathbb{H}^{n} .
$$

In particular, any $\mu$-eigenfunction of $\Delta$ with

$$
\mu=\left(a-\frac{1}{2}\right)^{2}-\left(\frac{n-1}{2}\right)^{2} \quad a=1, \ldots, k / 2
$$

is annihilated by $\square_{k}$.

Suppose now that we start with boundary data on $S^{n-1}$ that is focused at normal order $m$. That is, we take boundary data of some type $\mathbf{m} \ni m$, and assume the non- $m$ entries vanish. As noted in (35), the Casimir operator of $\mathfrak{s o}(n, 1)$ takes the value

$$
\mu_{n, k, m}:=\left(\frac{k-n-2 m}{2}+\frac{n-1}{2}\right)^{2}-\left(\frac{n-1}{2}\right)^{2}
$$

on the space of such data. Since the Casimir is represented by $\Delta$ on the interior and our extension operators are $G$-maps by conformal invariance, we have

$$
\mathcal{E}\left[\frac{k-n-2 m}{2}\right] \stackrel{\text { extension }}{\longrightarrow} \operatorname{Eig}\left(\Delta, \mu_{n, k, m}\right) .
$$

For this extension $\varphi \mapsto E \varphi$, it is natural to ask where our focused data $\varphi$ land upon restriction to $\mathbf{m}^{\prime}$-data on $S^{n}$. As noted in the discussion of (36), by looking at Casimir values again, the only possibility is to land in data focused at normal order $k-1-m$. Since the restriction operator is nonzero, it provides an inverse Poisson transform for a value of the $\mathfrak{a}^{*}$ parameter which is dual to the original one. In addition, the Knapp-Stein intertwining operator from (37),

$$
A_{k-1-2 m}: \mathcal{E}\left[\frac{k-n-2 m}{2}\right] \rightarrow \mathcal{E}\left[\frac{-k-n+2 m+2}{2}\right]
$$

has now been factored into Poisson and inverse Poisson transform operators (an extension and a restriction):

$$
A_{k-1-2 m}=\mathcal{P}_{(k-1-2 m) / 2}^{-1} \mathcal{P}_{-(k-1-2 m) / 2},
$$

where the subscripts denote rho-shifted $\mathfrak{a}^{*}$ parameters, and the factorization is through the interior eigenspace $\operatorname{Eig}\left(\Delta, \mu_{n, k, m}\right)$. 
There are known problems with the generalization of the classical process corresponding to (38) to the symmetric space case for more general orders; i.e., more general $q$ in $\mathcal{P}_{q}^{-1} \mathcal{P}_{-q}$. The values of $q$ giving problems are determined by the location of the zeros of the Harish-Chandra c-function; these may be read off from the Gindikin-Karpelevič formula (see, e.g., [15], Theorem 2.4). By [15], Remark 2.6, for $\mathrm{SO}_{0}(n, 1)$, there are no such zeros for $n$ odd; and for $n$ even, the zeros do not come into play for the values of $q$ involved in the above remarks. As a result, the intertwinors $A_{k-1-2 m}$ from (38) are indeed successfully factored into Poisson and inverse Poisson transforms.

We conclude with the following short remarks:

In the proof of Theorem 8.4, we have used the ellipticity of the target problem $\left((-1)^{k / 2} \square_{k}, \delta_{\mathbf{m}^{\prime}}^{\prime}\right)$, as well as that of the source problem $\left((-1)^{k / 2} \square_{k}, \delta_{\mathbf{m}}^{\prime}\right)$. If we run an analogous argument with non-complementary source and target problems, we may encounter the solution $\ell=m_{j}$ of (36). The operator $P_{k, \mathbf{m}, m_{j}, m_{j}}^{\prime}$ that we get in this case is simply the identity.

In the case of the sphere $S^{n-1}$ bounding the hemisphere $H^{n}$ used in the proof of Theorem $8.4, B^{2}$ is a differential operator with principal part $-\Delta_{S^{n-1}}$. Either $A_{k-1-2 m_{j}}$ or its inverse thus a composition of $B$ with $\mid k-$ $2-2 m_{j} \mid / 2$ differential operators of the form $B^{2}-p^{2}$.

In the conformally flat case,

$$
\underbrace{D_{A} \cdots D_{B}}_{k} u=X_{A} \cdots X_{B} \square_{k} u
$$

(see [29]). It follows from this and the definition of $\delta_{\ell}$ that the operators $P_{k, \mathbf{m}, m_{j}, \ell}$ for $\ell \geq k$ vanish on conformally flat structures.

In general, for a given odd integer $h$, we get several constructions of pseudo-differential operators with principal part $\left(-\Delta_{\Sigma}\right)^{h / 2}$, corresponding to the different values of $k>h$ allowed by Theorem 8.1.

Of course we also have conformally invariant operators with principal parts which are even powers of $\left(-\Delta_{\Sigma}\right)^{1 / 2}$, namely the $\square_{k}^{\Sigma}$ intrinsic to $\Sigma$. When these have trivial null space, their inverses are also conformally invariant pseudo-differential operators, and the range of orders for which we have conformally invariant, formally self-adjoint operators thus extends: If $n^{\Sigma}$ is odd, we get operators with principal part $\left(-\Delta_{\Sigma}\right)^{h / 2}$ for all even $h$, and for odd $|h|<n^{\Sigma}$. If $n^{\Sigma}$ is even, we get operators for all odd $h$, and for even $|h| \leq n^{\Sigma}$.

The operators described in Corollary 8.3 are heavily dependent on the choice of interior $M$. It may be possible to construct operators which are somewhat more intrinsic, by demanding that our choice of $M$ contain a given collar around the boundary, with a given conformal structure. This assures the preservation of invariant local constructions that use the boundary embedding. Under these circumstances, we would conjecture that the 
operators $P_{k, \mathbf{m}, m_{j}, k-1-m_{j}}^{\prime}$ still depend on the choice of $M$, but that any finite part of the expansion in Theorem 8.4, is independent of this choice, the coefficients being invariants of the boundary and boundary embedding.

Finally, in some situations, the following repackaging of the information in Theorem 8.1 might be useful. Suppose that both $\left(\square_{k}, \delta_{\mathbf{m}_{\mathrm{D}}}\right)$ and $\left(\square_{k}, \delta_{\mathbf{m}_{\mathrm{N}}}\right)$ have vanishing null space on $(M, \Sigma)$. Then one can collect the $P_{k, \mathbf{m}, m_{j}, \widetilde{m}_{i}}^{\prime}$ for $m_{j} \in \mathbf{m}_{\mathrm{D}}$ and $\widetilde{m}_{i} \in \mathbf{m}_{\mathrm{N}}$ into an operator $P_{\mathrm{D} \text { to N }}$ with a $(k / 2) \times(k / 2)$ block decomposition. For any $\psi \in \mathcal{E}\left[\frac{k-n}{2}\right]$ with $\square_{k} \psi=0$, this operator carries the conformal iterated Dirichlet data of $\psi$ to the conformal iterated Neumann data of $\psi$. There is a similarly defined operator $P_{\mathrm{N} \text { to D }}$, and in fact $P_{\mathrm{D} \text { to N }}$ and $P_{\mathrm{N} \text { to D }}$ are inverse to each other. One can combine these operators in the obvious way to get a natural (non-local) involution on the Cauchy data space. In the model case of the hemisphere with its bounding equator, the proof of Theorem 8.4 shows that, if we keep the ordering $\mathbf{m}_{\mathrm{D}}=$ $(0,2, \ldots, k-2)$ but reverse the ordering of the odd Cauchy data so that $\mathbf{m}_{\mathrm{N}}=(k-1, k-3, \ldots, 1)$, then the above-mentioned $(k / 2) \times(k / 2)$ block representations have non-zero entries only on the diagonal. As a result, in the case of general $(M, \Sigma)$, the graded leading symbols of these operators are supported on the diagonal. These operators are, moreover, formally self-adjoint: If $u, v$ are lists of conformal iterated Dirichlet data, then

$$
\int_{\Sigma}\left\{u P_{\mathrm{D} \text { to N }} v-\left(P_{\mathrm{D} \text { to N }} u\right) v\right\}=0
$$

since the expression on the left is a sum of terms of the form calculated (to be zero) in the proof of Theorem 8.5. A similar statement holds for $P_{\mathrm{N}}$ to D .

\section{References}

[1] T.N. Bailey, M.G. Eastwood and A.R. Gover, The Thomas structure bundle for conformal, projective and related structures, Rocky Mountain Journal of Math., 24 (1994), 1191-1217, MR 96e:53016, Zbl 828.53012.

[2] R. Baston, Verma modules and differential conformal invariants, J. Diff. Geom., 32 (1990) 851-898, MR 92a:58151, Zbl 732.53011.

[3] R. Baston and M. Eastwood, Invariant Operators, in "Twistors in Mathematics and Physics," London Mathematical Society Lecture Notes, 156, Cambridge University Press, (1990), 129-163, MR 92b:58247.

[4] H. Bateman, The transformation of the electrodynamical equations, Proc. London Math. Soc., 8 (1910), 223-264.

[5] W. Beckner, Sharp Sobolev inequalities on the sphere and the Moser-Trudinger inequality, Annals of Math., 138 (1993), 213-242, MR 94m:58232, Zbl 826.58042.

[6] T. Branson, Conformally covariant equations on differential forms, Commun. Partial Diff. Eq., 7 (1982), 393-431, MR 84g:58110, Zbl 532.53021.

[7] _ Differential operators canonically associated to a conformal structure, Math. Scand., 57 (1985), 293-345, MR 88a:58212, Zbl 596.53009. 
[8] _ Group representations arising from Lorentz conformal geometry, J. Funct. Anal., 74 (1987), 199-291, MR 90b:22016, Zbl 643.58036.

[9] _ Stein-Weiss operators and ellipticity, J. Funct. Anal., 151 (1997), 334-383, MR 99b:58219, Zbl 904.58054.

[10] _ Spectral theory of invariant operators, sharp inequalities, and representation theory, Supp. Rend. Circ. Matem. Palermo, 46 (1997), 29-54, MR 98k:58237, Zbl 884.58100.

[11] T. Branson, S.-Y.A. Chang and P. Yang, Estimates and extremals for zeta function determinants on four-manifolds, Commun. Math. Phys., 149 (1992), 241-262, MR 93m:58116, Zbl 761.58053.

[12] T. Branson and P. Gilkey, The asymptotics of the Laplacian on a manifold with boundary, Commun. Partial Diff. Eq., 15 (1990), 245-272, MR 90m:58201, Zbl 721.58052.

[13] , The functional determinant of a four-dimensional boundary-value problem, Trans. Amer. Math. Soc., 344 (1994), 479-531, MR 94k:58155, Zbl 811.58060.

[14] T. Branson, G. Ólafsson and B. Ørsted, Spectrum generating operators, and intertwining operators for representations induced from a maximal parabolic subgroup, J. Funct. Anal., 135 (1996), 163-205, MR 97g:22009, Zbl 841.22011.

[15] T. Branson, G. Ólafsson, and H. Schlichtkrull, Huyghens' principle in Riemannian symmetric spaces, Math. Ann., 301 (1995), 445-462, MR 97f:58128, Zbl 822.43002.

[16] E. Carlen and M. Loss, Competing symmetries, the logarithmic HLS inequality and Onofri's inequality on $S^{n}$, Geom. Anal. and Funct. Anal., 2 (1992), 90-104, MR 93b:58170, Zbl 754.47041.

[17] S.-Y.A. Chang and J. Qing, The zeta functional determinants on manifolds with boundary, I, II, J. Funct. Anal., 147 (1997), 327-362 and 363-399, MR 98f:58199, Zbl 914.58039, Zbl 914.58040.

[18] S.-Y.A. Chang and P. Yang, Extremal metrics of zeta function determinants on 4manifolds, Annals of Math., 142 (1995), 171-212, MR 96e:58034, Zbl 842.58011.

[19] A. Connes, Noncommutative Geometry, Academic Press, San Diego, 1994, MR 95j:46063, Zbl 818.46076.

[20] P.A.M. Dirac, Wave equations in conformal space, Ann. of Math., 37 (1936), 429-442, MR 58 \#32495, Zbl 014.08004.

[21] M.G. Eastwood, Notes on conformal differential geometry, Supp. Rend. Circ. Matem. Palermo, 43 (1996), 57-76, MR 98g:53021, Zbl 911.53020.

[22] M.G. Eastwood, Private communication.

[23] M.G. Eastwood and M. Singer, A conformally invariant Maxwell gauge, Phys. Lett., 107A (1985), 73-74, MR 86j:83031.

[24] J. Escobar, The Yamabe problem on manifolds with boundary, J. Diff. Geom., 35 (1992), 21-84, MR 93b:53030, Zbl 771.53017.

[25] _ Conformal deformation of a Riemannian metric to a scalar flat metric with constant mean curvature, Annals of Math., 136 (1992), 1-50; Addendum in Annals of Math., 139 (1994), 749-750, MR 95h:53053, Zbl 766.53033, Zbl 861.53036.

[26] C. Fefferman and C.R. Graham, Conformal invariants, in 'Elie Cartan et les mathématiques d'aujourd'hui', Astérisque, (1985), 95-116, MR 87g:53060, Zbl 602.53007. 
[27] P.B. Gilkey, Invariance Theory, the Heat Equation, and the Atiyah-Singer Index Theorem, CRC Press, Boca Raton, 1995, MR 98b:58156, Zbl 856.58001.

[28] A.R. Gover, Conformally invariant operators of standard type, Quart. J. Math., 40 (1989), 197-207, MR 90k:58254, Zbl 683.53063.

[29] _ Invariants and calculus for conformal geometry, to appear in Advances in Mathematics.

[30] _ Aspects of parabolic invariant theory, Supp. Rend. Circ. Matem. Palermo, Ser. II, Suppl., 59 (1999), 25-47, MR 2001a:58047.

[31] C.R. Graham, R. Jenne, L. Mason and G. Sparling, Conformally invariant powers of the Laplacian, I: Existence, J. Lond. Math. Soc., 46 (1992), 557-565, MR 94c:58226, Zbl 788.53011.

[32] G. Grubb, Functional Calculus of Pseudodifferential Boundary Problems, Second Edition, Birkhäuser, Boston, 1996, MR 96m:35001, Zbl 844.35002.

[33] S. Helgason, Geometric Analysis on Symmetric Spaces, American Mathematical Society, Providence, 1994, MR 96h:43009, Zbl 809.53057.

[34] L. Hörmander, The Analysis of Linear Partial Differential Operators III, SpringerVerlag, Berlin, 1985, MR 95h:35255, Zbl 601.35001.

[35] R. Kohn and M. Vogelius, Relaxation of a variational method for impedance computed tomography, Comm. Pure Appl. Math., 40 (1987), 745-777, MR 89h:35339, Zbl 659.49009.

[36] H. Kumano-go, Pseudo-differential Operators, MIT Press, Cambridge, 1974.

[37] J.L. Lions and E. Magenes, Non-homogeneous Boundary Value Problems and Applications, I, Springer-Verlag, Berlin, 1972, MR 50 \#2670, Zbl 227.35001.

[38] E. Onofri, On the positivity of the effective action in a theory of random surfaces, Commun. Math. Phys., 86 (1982), 321-326, MR 84j:58043, Zbl 506.47031.

[39] B. Osgood, R. Phillips and P. Sarnak, Extremals of determinants of Laplacians, J. Funct. Anal., 80 (1988), 148-211, MR 90d:58159, Zbl 653.53022.

[40] _ Compact isospectral sets of surfaces, J. Funct. Anal., 80 (1988), 212-234, MR 90d:58160, Zbl 653.53021.

[41] S. Paneitz, A quartic conformally covariant differential operator for arbitrary pseudoRiemannian manifolds, preprint, 1983.

[42] R. Penrose and W. Rindler, Spinors and Space-time, I, Cambridge University Press, 1984, MR 86h:83002, Zbl 538.53024.

[43] L. Peterson, Conformally covariant pseudo-differential operators, Diff. Geom. Appl., 13(2) (2000), 197-211, CMP 1783963.

[44] R. Riegert, A non-local action for the trace anomaly, Phys. Lett., 134B (1984), 56-60, MR 85g:81160.

[45] J. Slovák, Invariant operators on conformal manifolds, Lecture Notes, University of Vienna, 1992, archived at file://ftp.math.muni.cz.

[46] J. Sylvester and G. Uhlmann, Inverse boundary value problems at the boundary continuous dependence, Comm. Pure Appl. Math., 41 (1988), 197-219, MR 89f:35213, Zbl 632.35074.

[47] V. Wünsch, On conformally invariant differential operators, Math. Nachr., 129 (1986), 269-281, MR 88a:5820, Zbl 619.53008. 
Received November 9, 1999. The first author gratefully acknowledges support from US NSF grant INT-9724781. The second author gratefully acknowledges support from the Australian Department of Industry, Science \& Technology (Bilateral Collaboration Program), and from the Australian Research Council.

Department of Mathematics

THE UNIVERSITY OF IOWA

IOWA CiTy IA 52242 USA

E-mail address: branson@math.uiowa.edu

Department of Mathematics

The University of Auckland

Private Bag 92019, Auckland 1

NEW ZEALAND

E-mail address: gover@math.auckland.ac.nz 